\title{
The 55 Cancri system reassessed ${ }^{\star}$
}

\author{
V. Bourrier ${ }^{1}$, X. Dumusque ${ }^{1}$, C. Dorn ${ }^{2}$, G. W. Henry ${ }^{3}$, N. Astudillo-Defru ${ }^{4}$, J. Rey $^{1}$,

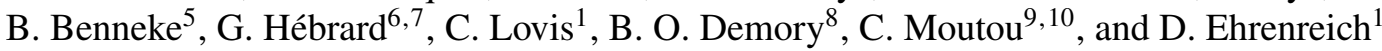

\author{
${ }^{1}$ Observatoire de l'Université de Genève, 51 chemin des Maillettes, 1290 Sauverny, Switzerland \\ e-mail: vincent.bourrier@unige.ch \\ ${ }^{2}$ Institut of Computational Sciences, University of Zurich, Winterthurerstrasse 190, 8057 Zurich, Switzerland \\ ${ }^{3}$ Center of Excellence in Information Systems, Tennessee State University, Nashville, TN 37209, USA \\ ${ }^{4}$ Departamento de Astronomía, Universidad de Concepción, Casilla 160-C Concepción, Chile \\ ${ }^{5}$ Département de Physique, Université de Montréal, Montreal H3T J4, Canada \\ ${ }^{6}$ CNRS, UMR 7095, Institut dAstrophysique de Paris, 98bis boulevard Arago, 75014 Paris, France \\ ${ }^{7}$ UPMC University of Paris 6, UMR 7095, Institut dAstrophysique de Paris, 98 bis boulevard Arago, 75014 Paris, France \\ ${ }^{8}$ Center for Space and Habitability, University of Bern, Sidlerstrasse 5, 3012 Bern, Switzerland \\ ${ }^{9}$ CFHT/CNRS, 65-1238 Mamalahoa Highway, Kamuela HI 96743, USA \\ ${ }^{10}$ Aix Marseille University, CNRS, LAM, Laboratoire d'Astrophysique de Marseille, Marseille, France
}

Received 4 April 2018 / Accepted 21 June 2018

\begin{abstract}
Orbiting a bright, nearby star the $55 \mathrm{Cnc}$ system offers a rare opportunity to study a multiplanet system that has a wide range of planetary masses and orbital distances. Using two decades of photometry and spectroscopy data, we have measured the rotation of the host star and its solar-like magnetic cycle. Accounting for this cycle in our velocimetric analysis of the system allows us to revise the properties of the outermost giant planet and its four planetary companions. The innermost planet 55 Cnce is an unusually close-in super-Earth, whose transits have allowed for detailed follow-up studies. Recent observations favor the presence of a substantial atmosphere yet its composition, and the nature of the planet, remain unknown. We combined our derived planet mass $\left(M_{\mathrm{p}}=8.0 \pm 0.3 M_{\text {Earth }}\right)$ with refined measurement of its optical radius derived from HST/STIS observations $\left(R_{\mathrm{p}}=1.88 \pm 0.03 R_{\text {Earth }}\right.$ over 530-750 nm) to revise the density of $55 \mathrm{Cnce}\left(\rho=6.7 \pm 0.4 \mathrm{~g} \mathrm{~cm}^{-3}\right)$. Based on these revised properties we have characterized possible interiors of $55 \mathrm{Cnce}$ using a generalized Bayesian model. We confirm that the planet is likely surrounded by a heavyweight atmosphere, contributing a few percents of the planet radius. While we cannot exclude the presence of a water layer underneath the atmosphere, this scenario is unlikely given the observations of the planet across the entire spectrum and its strong irradiation. Follow-up observations of the system in photometry and in spectroscopy over different time-scales are needed to further investigate the nature and origin of this iconic super-Earth.
\end{abstract}

Key words. stars: individual: $55 \mathrm{Cnc}$ - planets and satellites: fundamental parameters - planets and satellites: interiors stars: activity - techniques: radial velocities - techniques: photometric

\section{Introduction}

Visible to the naked eye, the G8 dwarf $55 \mathrm{Cnc}(V=5.95$, $d=12.3 \mathrm{pc}$; von Braun et al. 2011) hosts a diverse system of at least five exoplanets (Butler et al. 1997; Marcy et al. 2002; McArthur et al. 2004; Fischer et al. 2008; Dawson \& Fabrycky 2010), including a super-Earth orbiting in less than a day (55 Cnc e), a warm Jupiter possibly at the limit of atmospheric stability (55 Cnc b; Ehrenreich et al. 2012), and a gas giant with one of the longest known orbital periods (55 Cnc d, $\sim 15 \mathrm{yr}$ ). $55 \mathrm{Cnc}$, which is also in a binary system with an $\mathrm{M}$ dwarf at a projected separation of about 1060 au (Mugrauer et al. 2006), is one of the three brightest stars known to host a transiting super-Earth (between HD 219134, $V=5.5, d=6.5 \mathrm{pc}$; Motalebi et al. 2015; and HD 97658, $V=7.7, d=21.1 \mathrm{pc}$; Dragomir et al. 2013). Radial velocity measurements and transit observations of $55 \mathrm{Cnc}$ e have refined its mass $\left(8 M_{\text {Earth }}\right)$ and radius $\left(1.9 R_{\text {Earth }}\right)$

\footnotetext{
${ }^{\star}$ Individual APT photometric measurements for $55 \mathrm{Cnc}$ and its comparison stars, as well as RV measurements of $55 \mathrm{Cnc}$, are only available at the CDS via anonymous ftp to cdsarc.u-strasbg. fr (130.79.128.5) or via http://cdsarc.u-strasbg.fr/viz-bin/ qcat?J/A+A/619/A1
}

over the years (Fischer et al. 2008, 2017, Dawson \& Fabrycky 2010; Winn et al. 2011; Demory et al. 2011, 2012, 2016b; Gillon et al. 2012; Endl et al. 2012; Dragomir et al. 2014), up to the point where its bulk density can be measured precisely enough to constrain its interior structure. $55 \mathrm{Cnce}$ is one of the most massive members of the population of ultra-short period planets $(P \lesssim 1$ day) and stands on the upper radius side of the "evaporation valley" (Fulton et al. 2017) that might separate large super-Earths massive enough to retain $\mathrm{H} / \mathrm{He}$ envelopes with mass fractions of a few percent, and small rocky super-Earths with atmospheres that contribute negligibly to their size. The study of $55 \mathrm{Cnce}$ bulk and atmospheric composition is thus particularly important to our understanding of the formation and evolution of small, close-in planets.

The super-Earth 55 Cnc e has been the focus of detailed studies from the ultraviolet to the mid-infrared, yet its nature remains shrouded in mystery. Transit observations have shown that the planet does not harbour a hydrogen exosphere (Ehrenreich et al. 2012) or an extended water-rich atmosphere (Esteves et al. 2017), while the peculiar shape of its infrared phase curve is consistent with a heavyweight atmosphere (e.g., dust, metals, or water) rather than a magmatic surface with no atmosphere (Demory et al. 2016a; Angelo \& Hu 2017). High-resolution spectroscopy 
revealed changes in the transit depth of the optical sodium and singly-ionized calcium lines, possibly arising from variability in the structure of a putative exosphere (Ridden-Harper et al. 2016), while stellar emission lines in the far-ultraviolet showed variations that could trace strong interactions between $55 \mathrm{Cnce}$ and the stellar corona (Bourrier et al. 2018). Furthermore, Spitzer observations spanning three years have revealed significant temporal variability in the dayside-averaged thermal emission (Demory et al. 2016b), while MOST observations of the planet showed a significant decrease in the visible phasecurve amplitude between 2011 (Winn et al. 2011) and 2012 (Dragomir et al. 2014). All these elements point to the presence of a variable source of opacity in the atmosphere or at the surface of 55 Cnc e. Multiwavelength observations over different timescales are required to determine the nature of this source and characterize its variability. Understanding its origin further requires that we constrain the interior structure and composition of $55 \mathrm{Cnce}$, which is the objective of the present study.

We investigated the activity of the star in Sect. 2, and provide an updated velocimetric analysis of the planetary system in Sect. 3 that includes the stellar magnetic cycle effect for the first time. Ground-based and space-borne transit observations of 55 Cnce are presented in Sect. 4, and we combine our revised mass and radius measurements of the planet to model its interior in Sect. 5. We discuss the properties of $55 \mathrm{Cnc}$ e in Sect. 6, and we draw concludions from this updated analysis on the $55 \mathrm{Cnc}$ planetary system in Sect. 7.

\section{Analysis of stellar activity}

\subsection{Magnetic cycle of $55 \mathrm{Cnc}$}

We acquired 2243 good photometric observations of $55 \mathrm{Cnc}$ during 17 consecutive observing seasons between November 12, 2000 and April 17, 2017, all with the T8 $0.80 \mathrm{~m}$ automatic photoelectric telescope (APT) at Fairborn Observatory in southern Arizona. The T8 APT is one of several automated telescopes operated at Fairborn by Tennessee State University and is equipped with a two-channel precision photometer that uses a dichroic filter and two EMI 9124QB bi-alkali photomultiplier tubes to separate and simultaneously count photons in the Strömgren $b$ and $y$ passbands (Henry 1999).

We programmed the APT to make sequential brightness measurements of our program star $55 \mathrm{Cnc}(P: V=$ 5.96, $B-V=0.87, \mathrm{G} 8 \mathrm{~V})$ along with the three comparison stars HD $76572(C 1: V=6.25, B-V=0.47$, F6IV V), HD $77190(C 2: V=6.07, B-V=0.24$, A8V), and HD 79929 $(C 3: V=6.77, B-V=0.41, \mathrm{~F} 6 \mathrm{~V})$. From the raw counts in the two passbands, we computed six permutations of differential magnitudes from the four stars, namely, $P-C 1_{b y}, P-C 2_{b y}$, $P-C 3_{b y}, C 3-C 2_{b y}, C 3-C 1_{b y}$, and $C 2-C 1_{b y}$. We corrected the differential magnitudes for atmospheric extinction and transformed them to the Strömgren system. To improve our photometric precision, we combined the differential $b$ and $y$ observations into a single $(b+y) / 2$ passband, as indicated above with the subscript $b y$. Further information concerning our automated telescopes, precision photometers, and observing and data reduction techniques can be found in Henry (1995a,b, 1999) and Eaton et al. (2003) and references therein.

The first several years of our observations reveal all three comparison stars to be constant to the limit of our nightly precision ( $\sim 1$ milli-mags). We also find the seasonal means of comp stars $C 3$ and $C 2$ to be constant to the limit of our yearly precision ( $\sim 0.2$ milli-mags). However, comp $C 1$ exhibited

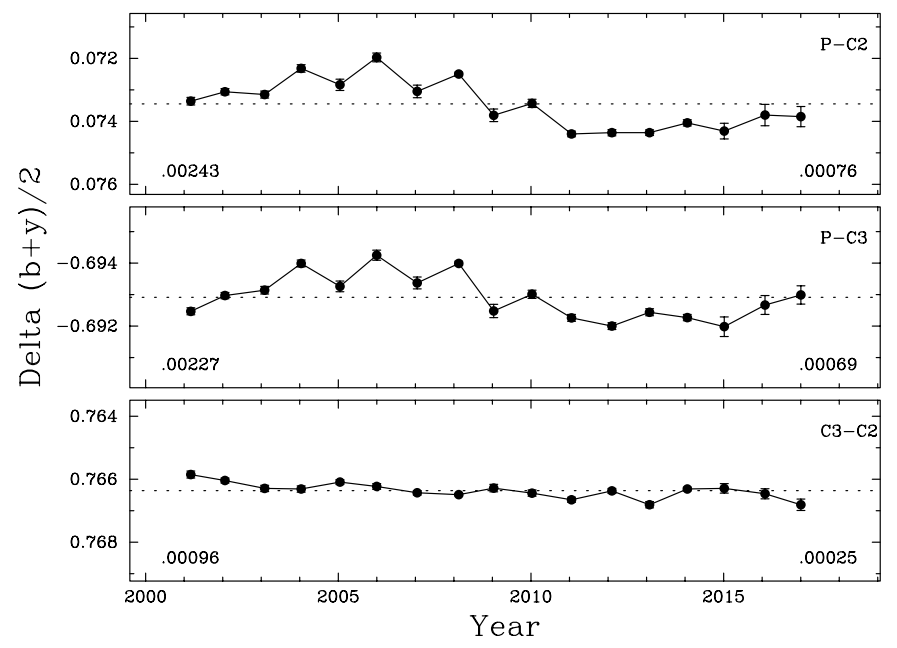

Fig. 1. APT optical photometry of $55 \mathrm{Cnc}$. First and second panels: seasonal mean differential magnitudes of $55 \mathrm{Cnc}(\mathrm{P})$ with respect to comparison stars $C 2$ and $C 3$. Third panel: seasonal mean differential magnitudes between comparison stars $C 3$ and $C 2$. The dotted lines show the grand means for all three panels. The total range of the observations and the standard deviation of the seasonal means from the grand mean are shown in the lower left and lower right of each panel, respectively. The $C 3-C 2$ differential magnitudes show excellent stability of $\pm 0.00025 \mathrm{mag}$, demonstrating that the variability in the $P-C 2$ and $P-C 3$ light curves is intrinsic to 55 Cnc.

year-to-year variability over a range of $\sim 3$ milli-mags or more. Therefore, we concentrated our photometric analyses on the differential magnitudes $P-C 3_{b y}, P-C 2_{b y}$, and $C 3-C 2_{b y}$, whose annual means are given in Table A.1 and plotted in Fig. 1 (individual measurements are available in electronic form at the $\mathrm{CDS}$ ). The differential magnitudes of $55 \mathrm{Cnc}$ with comp stars $C 3$ and $C 2$ revealed similar variations, with a peak-to-pea amplitude of $\sim 2$ milli-mag, significantly larger than the variability in $C 3-C 2_{b y}$. A sine curve fitted to $P-C 3_{b y}$ and $P-C 2_{b y}$ (Fig. 2) yields a period of about $14.4 \mathrm{yr}$ for this periodic variation, which we attribute to the magnetic cycle of $55 \mathrm{Cnc}$.

We further monitored $55 \mathrm{Cnc}$ from the ground, with five different spectrographs, yielding measurements of the $\mathrm{H} \alpha$ and $\mathrm{S}$ activity indexes over $\sim 20$ and $\sim 13 \mathrm{yr}$, respectively (see second and third panel in Fig. 2). Although the older observations with ELODIE are not extremely constraining, both indexes clearly show periodic variations arising from the magnetic cycle of $55 \mathrm{Cnc}$. The S-index data obtained by KECK HIRES highlights a solar-like cycle, with an amplitude of 0.024 (nearly twice that of the Sun which goes from $S=0.165-0.18$; e.g., Egeland et al. 2017 ) and a similar period, in this case $\sim 10.5 \pm 0.3 \mathrm{yr}$. This result is consistent with the cycle period of $12.6_{-1.0}^{+2.5}$ years estimated by Baluev (2015) from the analysis of RV data. We further derive a consistent period of $\sim 11.8 \mathrm{yr}$ from the $\mathrm{H} \alpha$ index; however, we note that the cycle has opposite phase in the S-index. Such an anti-correlation between $\mathrm{H} \alpha$ and $\mathrm{S}$-index have already been observed in other stars, however without a clear explanation (Gomes da Silva et al. 2014). We see a good correlation between the brightness variation in photometry and the S-index, which can be explained by a faculae to spot ratio increasing with activity, similar to what is observed in the Sun (Meunier et al. 2010a).

\subsection{Rotation period of $55 \mathrm{Cnc}$}

After correcting for the stellar magnetic cycle, we identified a sharp peak in the periodogram of $\mathrm{H} \alpha$ residuals at about 39 days 

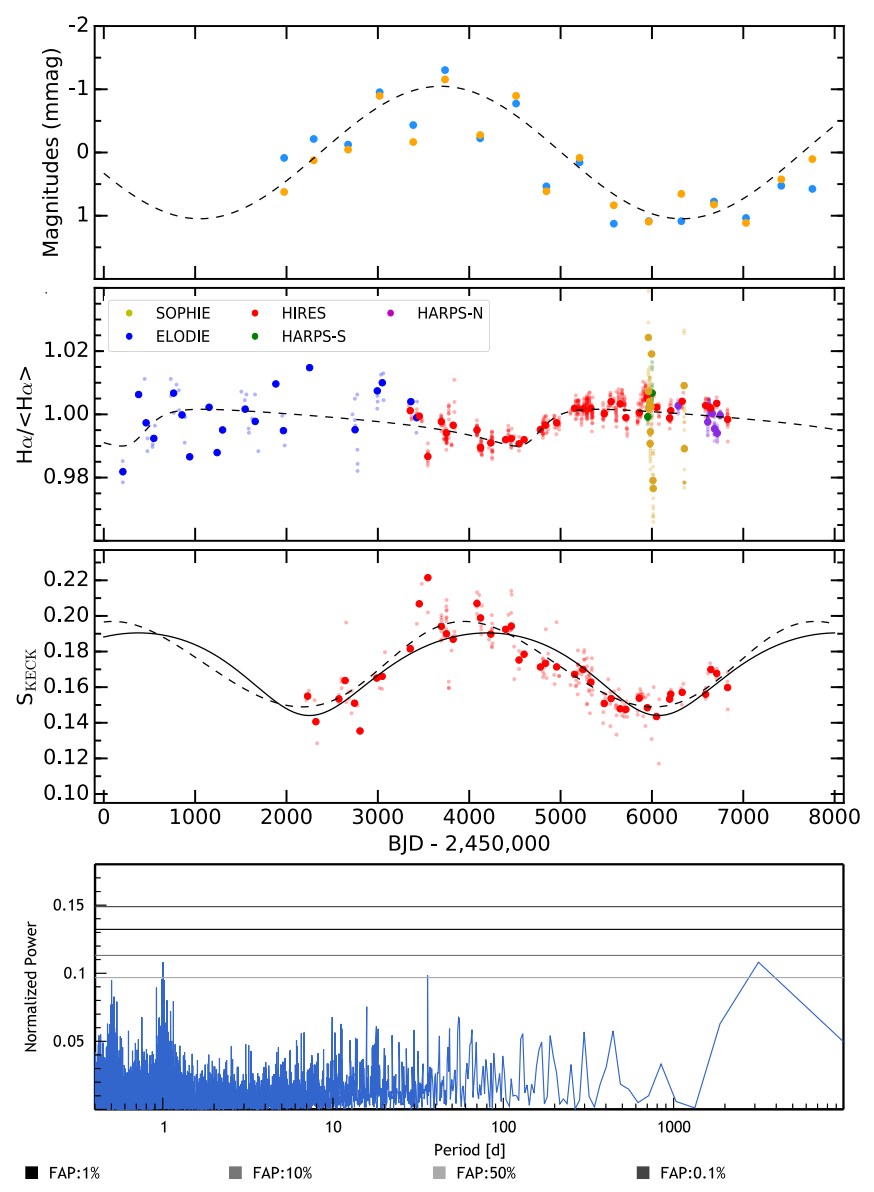

Fig. 2. Magnetic cycle of $55 \mathrm{Cnc}$. First panel: seasonal mean differential magnitudes of $55 \mathrm{Cnc}$ with comparison stars $C 2$ (blue) and $C 3$ (orange), with their best-fit sine function shown as a dashed black curve. Second panel: $55 \mathrm{Cnc} \mathrm{H} \alpha$ activity index and the best-fitted Keplerian to the data (dashed black curve). Third panel: $55 \mathrm{Cnc} \mathrm{S}$ activity index derived from KECK HIRES data. The continuous curve represents the best fitted Keplerian to those data, while the dashed curve corresponds to the best Keplerian fitted to the RVs to account for the RV effect of the stellar magnetic cycle (see Table 3 in Sect. 3). Fourth panel: periodogram of the $\mathrm{S}$-index residuals after removing the dashed curve seen in the third panel. The good match between the dashed curve and the continuous one in the third panel, as well as the absence of significant peaks in the periodogram of the S-index residuals, tells us that the extra Keplerian fitted to the RVs in Sect. 3 accounts well for the magnetic cycle effect.

(Fig. 3). A fit to the $\mathrm{H} \alpha$ residuals using a sine curve then yielded a period of $38.8 \pm 0.05$ days. The difference in Bayesian information criterion (BIC) between this model and a constant value is about 30 , confirming that we detect a significant signal in the activity index of $55 \mathrm{Cnc}$, which we associate with the stellar rotational modulation (Fig. 4).

Differential magnitudes of $55 \mathrm{Cnc}$ were corrected for longterm variations and normalized so that each observing season has the same mean. We first performed a frequency analysis based on least-squares sine fits and obtained a clear detection at $40.4 \pm 0.8$ days in season 9 , which we attribute to rotational modulation in the visibility of surface starspots (Fig. 4). We then compared the difference in BIC in each season between best-fits of a constant value and of a sine function initialized at the detected period. The error on the measurements was set to their overall standard deviation. In our models we included the best-fit transit function derived in Sect. 4.3 to avoid information

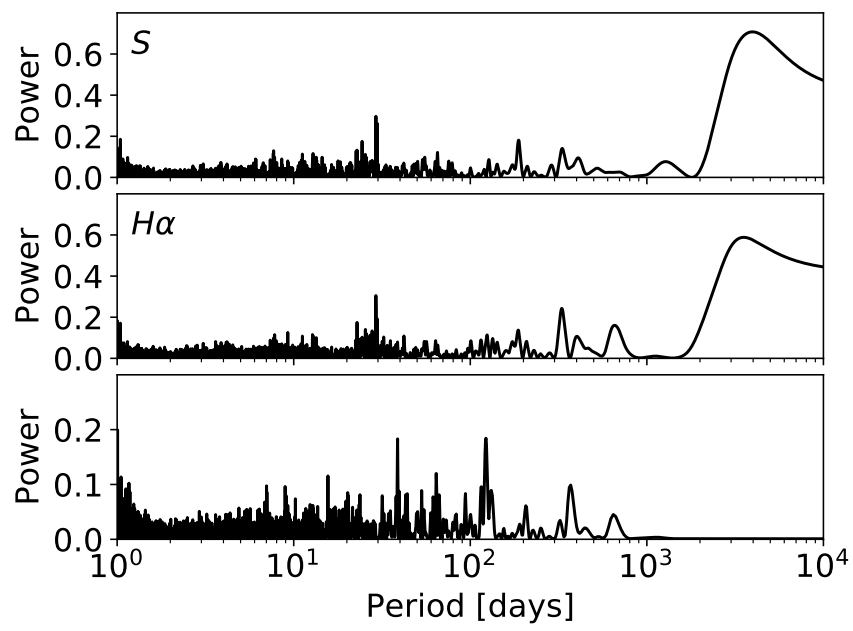

Fig. 3. Periodograms of $55 \mathrm{Cnc} \mathrm{S}$ and $\mathrm{H} \alpha$ activity indexes, showing power at the period of the stellar magnetic cycle. The bottom panel corresponds to $\mathrm{H} \alpha$ after correcting for the cycle.

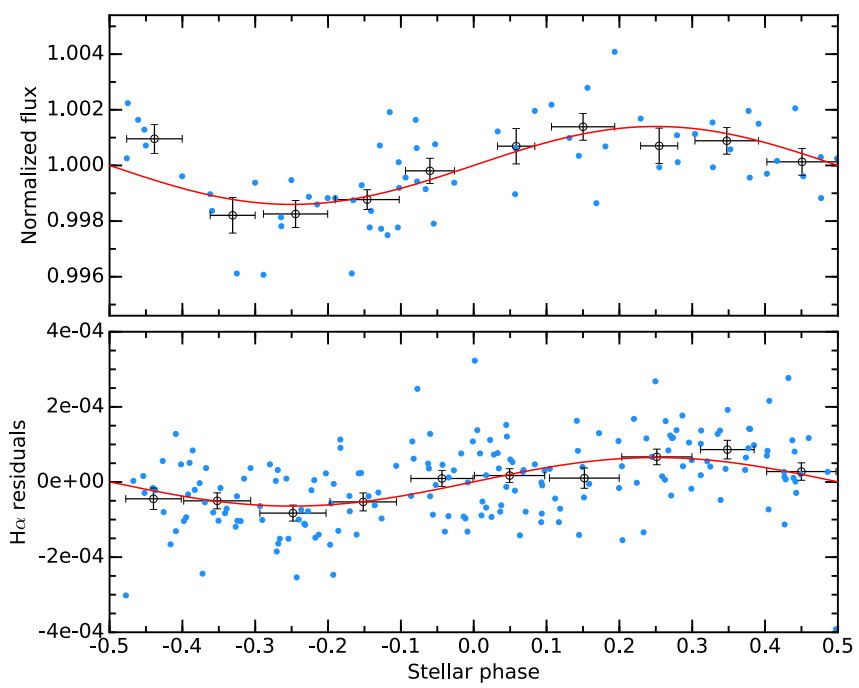

Fig. 4. Rotational modulation of $55 \mathrm{Cnc}$ in season 9 optical photometry (upper panel, $P_{*}=40.3$ days) and in the $\mathrm{H} \alpha$ index (lower panel, $P_{*}=38.8$ days). Data has been corrected for the stellar magnetic cycle and for the transit of $55 \mathrm{Cnce}$ (upper panel), then phase-folded to the periods detected in each dataset individually. Binned data points (black points) allow for a better comparison with the best-fit sine variations (red line).

loss. Sine functions yielded comparable or improved BIC values in seasons 5-10, and 17, which correspond to higher level of activity in the S-index. During a maximum activity phase, it is expected that more spots will be present on the solar surface, therefore, inducing a stronger photometric variability (see Fig. 2). Excluding Season 8, which exhibits a significantly larger periodicity at about 65 days, the other seasons show similar periods ranging from $\sim 35$ to 46 days (Table A.1). Their weighted mean, $40.04 \pm 0.39$ days, is consistent with the period measured in Season 9 and in the $\mathrm{H} \alpha$ residuals, and we adopted the latter as final best estimate for the rotation period of $55 \mathrm{Cnc}\left(P_{\text {rot }}=\right.$ $38.8 \pm 0.05$ days). This measurement is consistent with the period of $\sim 39$ days derived by Henry et al. (2000) from monitoring of the $\mathrm{Ca}$ II emission, and from previous estimates based on T8 APT photometry (42.7 \pm 2.5 days; Fischer et al. 2008).

We measure an average $\log \left(R_{\mathrm{HK}}^{\prime}\right)$ of -5.03 derived from KECK HIRES S-index data, which yields a rotation period 
Table 1. Properties of $55 \mathrm{Cnc}$ used and derived in our analysis.

\begin{tabular}{lccll}
\hline \hline Parameter & Symbol & Value & Unit & Reference \\
\hline Radius & $R_{\star}$ & $0.943 \pm 0.010$ & $R_{\text {sun }}$ & von Braun et al. (2011) \\
Mass & $M_{\star}$ & $0.905 \pm 0.015$ & $M_{\text {sun }}$ & von Braun et al. (2011) \\
Effective temperature & $T_{\text {eff }}$ & $5172 \pm 18$ & $\mathrm{~K}$ & Yee et al. (2017) \\
Surface gravity & $\log _{10}(g)$ & $4.43 \pm 0.02$ & & Yee et al. (2017) \\
Metallicity & {$[\mathrm{Fe} / \mathrm{H}]$} & $0.35 \pm 0.1$ & & Yee et al. (2017) \\
Limb-darkening coefficients & $u_{1}$ & $0.544 \pm 0.008$ & & This work \\
& $u_{2}$ & $0.186 \pm 0.004$ & & This work \\
Cycle period & $P_{\text {mag }}$ & $10.5 \pm 0.3$ & years & This work, derived from KECK HIRES S-index \\
Rotation period & $P_{\star}$ & $38.8 \pm 0.05$ & days & This work \\
Projected rotational velocity & $V \sin i_{\star}$ & $<1.23 \pm 0.01$ & $\mathrm{~km} \mathrm{~s}^{-1}$ & This work \\
\hline
\end{tabular}

of $49.8 \pm 4.8$ days from the empirical relations in both Noyes et al. (1984) and Mamajek \& Hillenbrand (2008; we caution that $55 \mathrm{Cnc}$ is at the edge of the sample used by both authors). López-Morales et al. (2014) obtained similar results from HARPS and HARPS-N spectra, while Brewer et al. (2016) derived $\log \left(R_{\mathrm{HK}}^{\prime}\right)=-4.98$ from a subset of the data we have for KECK HIRES, which yields a rotation period of $46.4 \pm$ 4.8 days. Our $\log \left(R_{\mathrm{HK}}^{\prime}\right)$ further yields an age of $\sim 8.6 \pm 1.0 \mathrm{Gyr}$ from the relations in Mamajek \& Hillenbrand (2008), compatible with the value of about $8 \mathrm{Gyr}$ derived by Brewer et al. (2016) from isochrone fitting, but lower than the ages derived via a similar method by von Braun et al. $(2011 ; 10.2 \pm 2.5 \mathrm{Gyr})$ and Yee et al. $\left(2017 ; 12.6_{-2.3}^{+2.9} \mathrm{Gyr}\right)$. While the $\operatorname{low} \log \left(R_{\mathrm{HK}}^{\prime}\right)$ shows that $55 \mathrm{Cnc}$ is an old and chromospherically inactive star, we note that it shows temporal variations at X-ray and far-UV wavelengths that trace variability in the upper chromosphere and corona (Ehrenreich et al. 2012, Bourrier et al. 2018).

Our measurement of the stellar rotation period was further used to set an upper limit on the projected stellar rotational velocity, $V \sin i_{\star}<\left(2 \pi R_{\star}\right) / P_{\star}=1.23 \pm 0.01 \mathrm{~km} \mathrm{~s}^{-1}$. This value is consistent with the velocity derived from stellar line broadening by Brewer et al. (2016; $\left.1.7 \pm 0.5 \mathrm{~km} \mathrm{~s}^{-1}\right)$ and with the upper limit obtained by López-Morales et al. (2014) from the nondetection of the Rossiter-McLaughlin effect of $55 \mathrm{Cnce}$ $\left(0.2 \pm 0.5 \mathrm{~km} \mathrm{~s}^{-1}\right)$. It is, however, about $2.3 \sigma$ lower than the previous velocity derived from stellar line broadening by Valenti \& Fischer $\left(2005 ; 2.4 \pm 0.5 \mathrm{~km} \mathrm{~s}^{-1}\right)$ than the velocity derived by Bourrier \& Hébrard (2014) from the possible detection of the Rossiter-McLaughlin effect $\left(3.3 \pm 0.9 \mathrm{~km} \mathrm{~s}^{-1}\right)$.

\section{Velocimetric analysis of the 55 Cnc system}

The radial velocity (RV) analysis presented here combines all the public data of $55 \mathrm{Cnc}$ in addition to unpublished out-of-transit SOPHIE data. Therefore, we use the 343 data points from the Tull and HRS spectrograph (Endl et al. 2012), the $250 \mathrm{RV}$ measurements from Lick Observatory (Fischer et al. 2008), the 629 data points from KECK HIRES (Butler et al. 2017), the 292 spectra from HARPS and HARPS-N (López-Morales et al. 2014), and 38 data points from SOPHIE. Those $1552 \mathrm{RV}$ measurements were binned over a timescale of $30 \mathrm{~min}$, to average out stellar oscillations and high-frequency granulation (Dumusque et al. 2011b). Some observations were taken during the transit of $55 \mathrm{Cnce}$, which could affect the RVs due to a Rossiter-McLaughlin effect (Bourrier \& Hébrard 2014). We note however, that the detection of the Rossiter-McLaughlin effect induced by $55 \mathrm{Cnce}$ is called into question by López-Morales et al. (2014), and even if present, its effect can be neglected in this analysis due to an amplitude smaller than $0.5 \mathrm{~m} \mathrm{~s}^{-1}$ (Bourrier \& Hébrard 2014).

To update the orbital parameters of the known planets orbiting $55 \mathrm{Cnc}$ using all the published data plus the few additional ones taken with SOPHIE, we performed a fit using the tools available on the Data \& Analysis Center for Exoplanet (DACE ${ }^{1}$ ). Besides the five planets already known, we have added an extra Keplerian to take into account the effect of the stellar magnetic cycle. As discussed in Dumusque et al. (2011a) and Meunier et al. (2010b), such a magnetic cycle can have a correlated counter part in RVs, with an estimated amplitude of $\sim 12 \mathrm{~m} \mathrm{~s}^{-1}$ (Lovis et al. 2011) considering the stellar properties of $55 \mathrm{Cnc}\left(B-V=0.87, T_{\text {eff }}=5172, \mathrm{Fe} / \mathrm{H}=0.35\right.$; see Table 1).

All the parameters fitted with their priors can be found in Table 2. The planet orbital parameters were initialized by first searching for the planetary signals in a generalized LombScargle (GLS) periodogram (Zechmeister \& Kürster 2009) and then finding the best solution using a Levenberg-Marquardt algorithm. Regarding the Keplerian to account for the effect of the magnetic cycle in the RVs, its initial parameters were set by first fitting the KECK HIRES S-index with a sinusoidal and imposing an amplitude of $12 \mathrm{~m} \mathrm{~s}^{-1}$, as estimated. Uniform priors were set for all the parameters, except the stellar mass for which we adopted a Gaussian prior $M_{55 \mathrm{Cnc}}=$ $\mathcal{N}(0.905,0.015) M_{\odot}$ from von Braun et al. (2011) and the period and transit time of $55 \mathrm{Cnc}$, for which we also adopted Gaussian priors derived from Spitzer observations $P_{55 \text { Cnce }}=$ $\mathcal{N}(0.73654627800,0.0000018477)$ days and $T_{\text {transit, } 55 \mathrm{Cnce}}=$ $\mathcal{N}\left(55733.0058594,1.464810^{-3}\right)$ days (B. Demory, priv. comm.). Starting from those initial conditions and priors, we ran the MCMC algorithm available on DACE with $2 \times 10^{6}$ iterations.

After removing the first $5 \times 10^{5}$ iterations to reject the burn-in period and applying a thining of 384 to remove all correlation within the chains, the result of the MCMC can be found in Table A.2. The median of the posterior of the parameters of interest with $68.3 \%$ confidence intervals are shown in Table 3. The best fit using the median of all the marginalized posteriors, as well as the RV residuals and corresponding GSL periodogram is presented in Fig. 5. As we can see, only one signal at 12.9 days has a $p$-value smaller than $1 \%$. The significance of this signal is not high enough to consider a potential extra planet in the system, and with planet b at 14.7 days, this seems unlikely. This signal is very likely an harmonic of the stellar

$\overline{1}$ http://dace.unige.ch 
Table 2. List of parameters probed by the MCMC.

\begin{tabular}{|c|c|c|c|}
\hline$\underline{\text { Parameters }}$ & Units & Priors & Description \\
\hline \multicolumn{4}{|l|}{ Parameters probed by MCMC } \\
\hline$M_{\star}$ & $\left(M_{\odot}\right)$ & $\mathcal{N}(0.915,0.015)$ & Stellar mass of $55 \mathrm{Cnc}$ \\
\hline$\sigma_{J I T}$ & $\left(\mathrm{~m} \mathrm{~s}^{-1}\right)$ & $\mathcal{U}$ & Stellar jitter \\
\hline$\sigma_{\text {(HRS, Tull, Lick, KECK, HARPN, HARPS, SOPHIE) }}$ & $\left(\mathrm{m} \mathrm{s}^{-1}\right)$ & $\mathcal{U}$ & Instrumental jitter \\
\hline$\gamma_{(\mathrm{HRS}, \text { Tull, Lick, KECK, HARPN, HARPS, SOPHIE) }}$ & $\left(\mathrm{km} \mathrm{s}^{-1}\right)$ & $\mathcal{U}$ & Constant velocity offset \\
\hline$P_{55 \mathrm{Cnce}}$ & (days) & $\mathcal{N}\left(0.73654627800,1.847710^{-6}\right)$ & Period \\
\hline $\log (P)(55 \mathrm{Cnc} b, \mathrm{c}, \mathrm{f}, \mathrm{d}$, magn. cycle $)$ & $\log$ (days) & $\mathcal{U}$ & Logarithm of the period \\
\hline $\log (K)(55$ Cnc e, b, c, f, d, magn. cycle) & $\log \left(\mathrm{m} \mathrm{s}^{-1}\right)$ & $\mathcal{U}$ & Logarithm of the RV semi-amplitude \\
\hline$\sqrt{e} \cos \omega(55$ Cnc e, b, c, f, d, magn. cycle) & - & $\mathcal{U}$ & \\
\hline$\sqrt{e} \sin \omega(55$ Cnc e, b, c, f, d, magn. cycle $)$ & - & $\mathcal{U}$ & \\
\hline$T_{\text {transit }}(55 \mathrm{Cnc}$ ) $)$ & (d) & $\mathcal{N}\left(55733.0058594,1.464810^{-3}\right)$ & Transit time for $55 \mathrm{Cnce}$ \\
\hline$\lambda_{0}(55 \mathrm{Cnc} b, \mathrm{c}, \mathrm{f}, \mathrm{d}$, magn. cycle $)$ & $(\mathrm{deg})$ & $\mathcal{U}$ & Mean longitude \\
\hline
\end{tabular}

Physical Parameters derived from the MCMC posteriors (not probed)

\begin{tabular}{lcll}
$P$ & $(\mathrm{~d})$ & - & Orbital period \\
$K$ & $\left(\mathrm{~m} \mathrm{~s}^{-1}\right)$ & - & RV semi-amplitude \\
$e$ & - & - & Orbital eccentricity \\
$\omega$ & $(\mathrm{deg})$ & - & Argument of periastron \\
$T_{C}$ & $(\mathrm{~d})$ & - & Time of transit or inferior conjunction \\
$a$ & $(\mathrm{AU})$ & - & Semi-major axis of the relative orbit \\
$M$ & $\left(M_{\text {Jup }}\right)$ & - & Mass relative to Jupiter (when the inclination $i$ is known) \\
$M$ & $\left(M_{\text {Earth }}\right)$ & - & Mass relative to Earth (when the inclination $i$ is known) \\
$M \sin i$ & $\left(M_{\text {Jup }}\right)$ & - & Minimum mass relative to Jupiter \\
$M \sin i$ & $\left(M_{\text {Earth }}\right)$ & - & Minimum mass relative to Earth \\
\hline
\end{tabular}

Notes. The symbols $\mathcal{U}$ and $\mathcal{N}$ used for the priors definition stands for uniform and normal distributions, respectively.

rotation period, as it corresponds to a third of its value (38.8 days, Sect. 2.2). The RV of each planet and the magnetic cycle folded in phase are shown in Fig. 6 (individual measurements from each instrument are available in electronic form at the CDS).

We note that the planetary parameters that we derive are close to the ones published in Endl et al. (2012) and Fischer (2017), however not always compatible within $3 \sigma$. The most significant difference comes from planet $\mathrm{d}$, the outermost planet in the system that we found at a period of $5574_{-89}^{+94}$ days, while it was estimated at $4909 \pm 30$ and $5285 \pm 4.5$ days in Endl et al. (2012) and Fischer (2017), respectively. This large difference can be explained by the fact that we take into account the nonnegligible $15 \mathrm{~m} \mathrm{~s}^{-1}$ effect of the magnetic cycle in our analysis, which was not done in the past. To make sure that the Keplerian we fitted to account for the magnetic cycle indeed take into account this effect and does not fit any spurious signal in the RV residuals, we show the fitted signal on top of the KECK HIRES S-index in Fig. 2 (third panel, dashed line). We only adjusted the amplitude as RV and S-index are not on the same scale. The strong correlation between this Keplerian and the S-index variation and no significant signal in the S-index residuals after removing this Keplerian (Fig. 2 fifth panel) tell us that this extra component takes correctly into account the RV effect induced by the magnetic cycle of $55 \mathrm{Cnc}$. Other significant differences can be seen for the eccentricity of planet e and f, Fischer (2017) reporting significant eccentricities of $0.22 \pm 0.05$ and $0.27 \pm$ 0.05 , respectively. Our solution converges to smaller eccentricities of $0.05 \pm 0.03$ and $0.08 \pm 0.05$, compatible within 0 at $2 \sigma$. The smaller eccentricity for planet $\mathrm{e}$ is in agreement with orbital circularization, which is expected for such short orbital periods.

\section{Photometric analysis of 55 Cnce transit}

\subsection{Extraction of HST/STIS $1 D$ spectra}

Transit observations of $55 \mathrm{Cnce}$ were obtained with the lowresolution G750L grating of the Space Telescope Imaging Spectrograph (STIS) spectrograph onboard the Hubble Space Telescope (HST; PI: Benneke, GO program 13665). This grating covers the wavelength range $524-1027 \mathrm{~nm}$ with a dispersion of $4.92 \AA$ per pixel. Three visits were obtained on 2014, 30 October (Visit $A_{\text {STIS }}$ ), 2015, 10 May (Visit B BTIS), and 2015, 22 May (Visit C $_{\text {STIS }}$ ), each visit consisting in five HST orbits. Observations were taken in ACCUM mode, yielding 28 subexposures the first orbit of all visits, and 34 (Visit A $_{\text {STIS }}$ ) or 41 (Visits $\mathrm{B}_{\mathrm{STIS}}$ and $\mathrm{C}_{\mathrm{STIS}}$ ) subexposures in subsequent orbits. All subexposures have a duration of $36 \mathrm{~s}$. The last orbit in each visit was taken as a fringe flat intended to help correcting the near-infrared portion $(>750 \mathrm{~nm})$ of the spectra from CCD fringing. However, observations were oversaturated and we do not know how that can affect the correction for the fringing effect. To avoid any potential bias in the derivation of $55 \mathrm{Cnc}$ e transit depth, we thus discarded the region of the G750L spectra affected by fringing redward of $750 \mathrm{~nm}$.

Oversaturation led charges to bleed far along the detector columns. They were retrieved using a custom rectangular extraction aperture that we applied to the flat-fielded science files (FLT) output by the STIS calibration pipeline CALSTIS (e.g. Demory et al. 2015). Some images have a height of 500 pixels along the cross-dispersion axis, instead of 1024 pixels, but we used the same aperture for all images to extract the spectra in a consistent manner. The width of the aperture covers the full length of the dispersion axis (1024 pixels) and 
Table 3. Best-fitted solution for the planetary system orbiting $55 \mathrm{Cnc}$.

\begin{tabular}{|c|c|c|c|c|c|c|c|}
\hline Param. & Units & $55 \mathrm{Cnce}$ & $55 \mathrm{Cncb}$ & $55 \mathrm{Cncc}$ & $55 \mathrm{Cncf}$ & Magnetic cycle & $55 \mathrm{Cnc} \mathrm{d}$ \\
\hline$P$ & (d) & $0.73654737_{-1.4410^{-6}}^{+1.3010^{-6}}$ & $14.6516_{-0.0001}^{+0.0001}$ & $44.3989_{-0.0043}^{+0.0042}$ & $259.88_{-0.29}^{+0.29}$ & $3822.4_{-77.4}^{+76.4}$ & $5574.2_{-88.6}^{+93.8}$ \\
\hline$K$ & $\left(\mathrm{~m} \mathrm{~s}^{-1}\right)$ & $6.02_{-0.23}^{+0.24}$ & $71.37_{-0.21}^{+0.21}$ & $9.89_{-0.22}^{+0.22}$ & $5.14_{-0.25}^{+0.26}$ & $15.2_{-1.8}^{+1.6}$ & $38.6_{-1.4}^{+1.3}$ \\
\hline$e$ & & $0.05_{-0.03}^{+0.03}$ & $0.00_{-0.01}^{+0.01}$ & $0.03_{-0.02}^{+0.02}$ & $0.08_{-0.04}^{+0.05}$ & $0.17_{-0.04}^{+0.04}$ & $0.13_{-0.02}^{+0.02}$ \\
\hline$\omega$ & $(\operatorname{deg})$ & $86.0_{-33.4}^{+30.7}$ & $-21.5_{-89.8}^{+56.9}$ & $2.4_{-49.2}^{+43.1}$ & $-97.6_{-51.3}^{+37.0}$ & $174.7_{-14.1}^{+16.6}$ & $-69.1_{-7.9}^{+9.1}$ \\
\hline$T_{C}$ & (d) & $55733.0060_{-0.0014}^{+0.0014}$ & $55495.587_{-0.016}^{+0.013}$ & $55492.02_{-0.42}^{+0.34}$ & $55491.5_{-4.8}^{+4.8}$ & $55336.9_{-50.6}^{+45.5}$ & $56669.3_{-76.5}^{+83.6}$ \\
\hline$a$ & $(\mathrm{AU})$ & $0.0154_{-0.0001}^{+0.0001}$ & $0.1134_{-0.0006}^{+0.0006}$ & $0.2373_{-0.0013}^{+0.0013}$ & $0.7708_{-0.0044}^{+0.0043}$ & - & $5.957_{-0.071}^{+0.074}$ \\
\hline$M$ & $\left(M_{\mathrm{Jup}}\right)$ & $0.0251_{-0.0010}^{+0.0010}$ & - & - & - & - & - \\
\hline$M$ & $\left(M_{\text {Earth }}\right)$ & $7.99_{-0.33}^{+0.32}$ & - & - & - & - & - \\
\hline$M \sin i$ & $\left(M_{\mathrm{Jup}}\right)$ & - & $0.8036_{-0.0091}^{+0.0092}$ & $0.1611_{-0.0040}^{+0.0040}$ & $0.1503_{-0.0076}^{+0.0076}$ & - & $3.12_{-0.10}^{+0.10}$ \\
\hline$M \sin i$ & $\left(M_{\text {Earth }}\right)$ & - & $255.4_{-2.9}^{+2.9}$ & $51.2_{-1.3}^{+1.3}$ & $47.8_{-2.4}^{+2.4}$ & - & $991.6_{-33.1}^{+30.7}$ \\
\hline$\sigma_{(\mathrm{O}-\mathrm{C}) \text { HARPN }}$ & $\left(\mathrm{m} \mathrm{s}^{-1}\right)$ & & & 1.20 & & & \\
\hline$\sigma_{(\mathrm{O}-\mathrm{C}) \text { HARPS }}$ & $\left(\mathrm{m} \mathrm{s}^{-1}\right)$ & & & 0.81 & & & \\
\hline$\sigma_{(\mathrm{O}-\mathrm{C}) \mathrm{HRS}}$ & $\left(\mathrm{m} \mathrm{s}^{-1}\right)$ & & & 4.38 & & & \\
\hline$\sigma_{(\mathrm{O}-\mathrm{C}) \mathrm{KECK}}$ & $\left(\mathrm{m} \mathrm{s}^{-1}\right)$ & & & 3.58 & & & \\
\hline$\sigma_{(\mathrm{O}-\mathrm{C}) \text { LICK }}$ & $\left(\mathrm{m} \mathrm{s}^{-1}\right)$ & & & 6.61 & & & \\
\hline$\sigma_{(\mathrm{O}-\mathrm{C})}$ SOPHIE & $\left(\mathrm{m} \mathrm{s}^{-1}\right)$ & & & 2.02 & & & \\
\hline$\sigma_{(\mathrm{O}-\mathrm{C}) \mathrm{TULL}}$ & $\left(\mathrm{m} \mathrm{s}^{-1}\right)$ & & & 4.89 & & & \\
\hline$\sigma_{(\mathrm{O}-\mathrm{C}) \text { all }}$ & $\left(\mathrm{m} \mathrm{s}^{-1}\right)$ & & & 4.33 & & & \\
\hline $\log$ (Post) & & & & $-2285.8_{-5.1}^{+4.5}$ & & & \\
\hline
\end{tabular}

Notes. For each parameter, the median of the posterior is considered, with error bars computed from the MCMC marginalized posteriors using a $68.3 \%$ confidence interval. $\sigma_{(O-C) X}$ corresponds to the standard deviation of the residuals around this best solutions for instrument X, and $\sigma_{(O-C) \text { all }}$ the weighted standard deviation for all the data. All the parameters probed by the MCMC can be found in Table A.2.
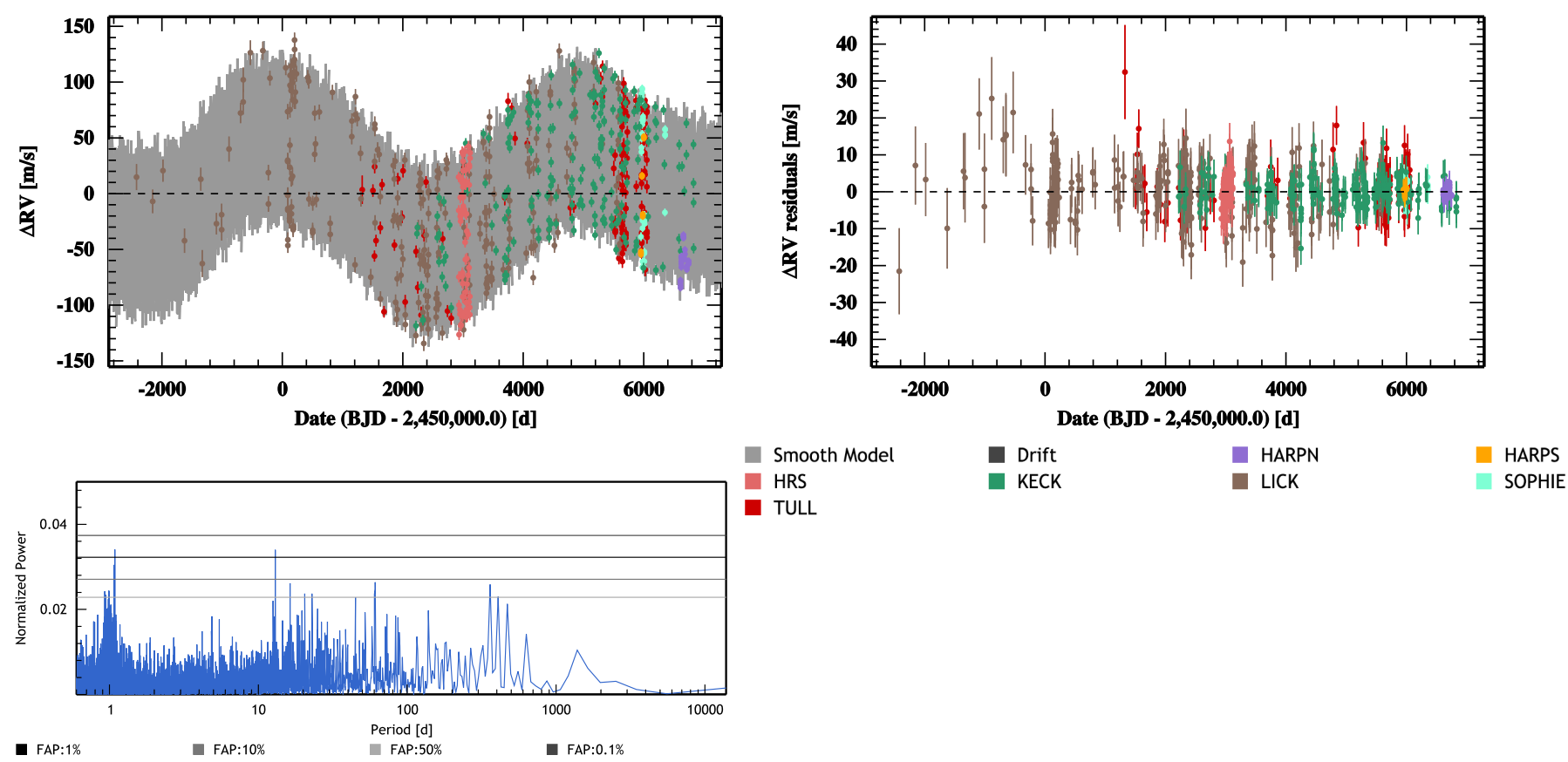

SOPHIE

Fig. 5. Top left panel: $1552 \mathrm{RV}$ measurements binned over a timescale of $30 \mathrm{~min}$ with the best model overplotted. This model is obtained by taking the median of each marginalized posterior after our MCMC run. Top right panel: RV residuals after removing the best model. Bottom left panel: GLS periodogram of the RV residuals including false-alarm probability detection thresholds. 

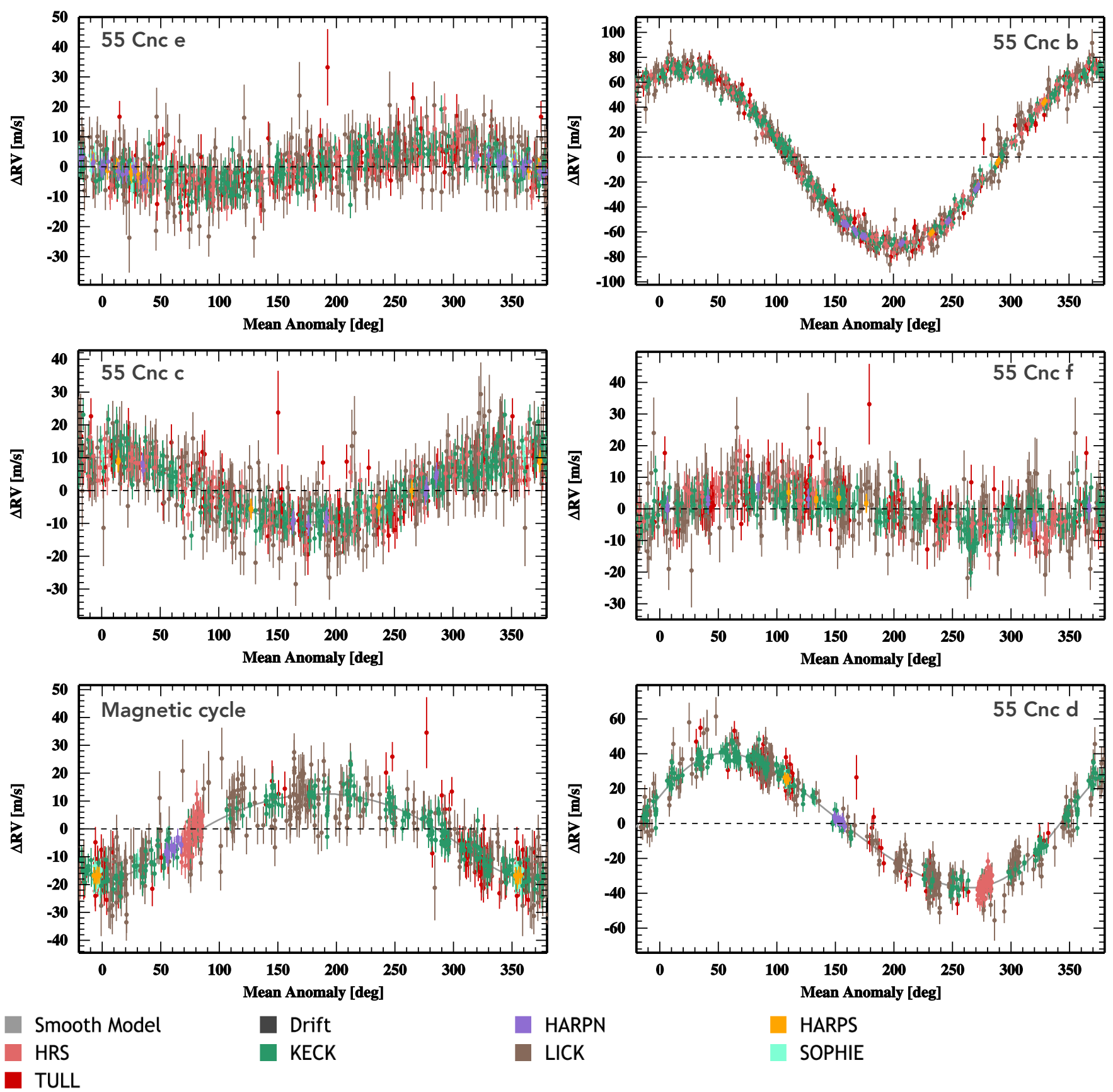

Fig. 6. RV of each planet and the magnetic cycle, folded in phase using the best-fitted orbital periods. The signals are ordered with orbital periods, therefore, from left to right and top to bottom, we have planet e, b, c, f, the magnetic cycle, and planet $\mathrm{d}$.

its height was set to 390 pixels to retrieve as many charges as possible while allowing for the background spectrum to be measured. The background was averaged within two regions, 40 pixels in height, and starting 8 pixels above and below the edges of the extraction aperture. We removed cosmics and bad pixels from the background spectrum using median filtering with a running window. It was then fitted with a fifth-order polynomial function in each exposure, which was used to correct the extracted stellar spectra. We attributed to the corrected spectra the wavelength tables issued by the CALSTIS pipeline, and aligned the spectra in each visit by cross-correlating them with their overall mean over the visit. We then compared each spectrum with the average of the other spectra in the same HST orbit, identifying pixels with count rates larger than five times the standard deviation. We attributed to these pixels the count rate from the averaged spectrum. This operation was repeated twice, and spectra were carefully checked for any residual spurious features. We removed the bluest 10 pixels in all spectra, as we found they were varying significantly over each visit. All analyses hereafter are performed on the spectra integrated over the range remaining after excluding these pixels $(531-750 \mathrm{~nm})$.

\subsection{Analysis of HST/STIS transit light curve}

STIS observations are affected by variations in the telescope throughput caused by thermal variations that HST experiences during each orbit (e.g., Brown et al. 2001; Sing et al. 2008; 


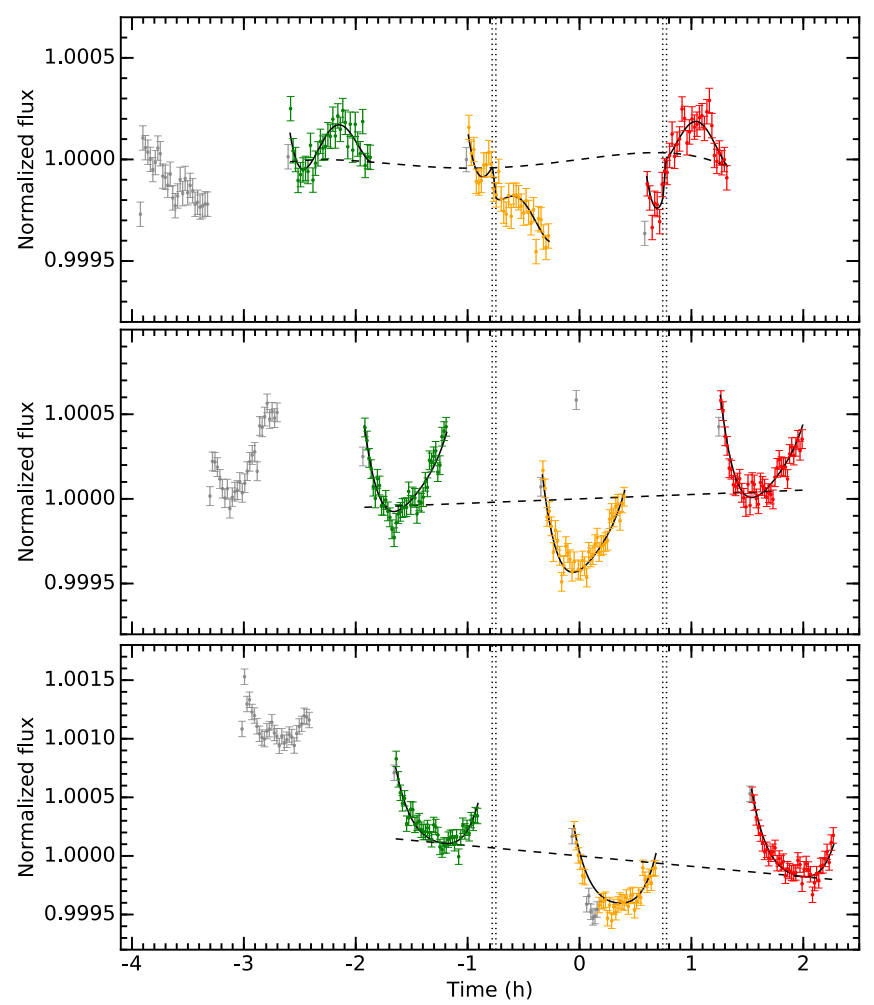

Fig. 7. STIS spectra of $55 \mathrm{Cnc}$ integrated over the visible band, and plotted as a function of time relative to the transit of $55 \mathrm{Cnc}$ e (vertical dotted lines show the beginning and end of ingress and egress of the transit). The solid black line is the best-fit model to the data, which includes the breathing and long-term flux variations, and the transit. The black dashed line is the model contribution to the long-term variations. Visit $\mathrm{A}_{\mathrm{STIS}}, \mathrm{B}_{\mathrm{STIS}}$, and $\mathrm{C}_{\mathrm{STIS}}$ are plotted from top to bottom. The second, third, and fourth orbits in each visit are colored in green, orange, and red. Exposures excluded from the fit are plotted in gray.

Evans et al. 2013). This "breathing" effect modifies the flux balance within an HST orbit, and is known to be achromatic for a given STIS grating. Our observations display the typical behavior of optical gratings (Fig. 7), with the first orbit showing different flux level and breathing trend than the other orbits. In addition, the first exposure in each orbit shows a significantly lower flux (see e.g., Huitson et al. 2012; Sing et al. 2013). First orbits and first exposures were subsequently excluded from our analysis. We also identified long-term variations in each visit that could be linked to instrumental stability or variability in the intrinsic stellar flux (Fig. 7). For all visits we find that a fourth-order polynomial function of HST phase was sufficient to describe the breathing variations (Fig. 8), in agreement with previous studies (eg Sing et al. 2008; Demory et al. 2015). The first-order term of this polynomial was found to be unnecessary in Visit $\mathrm{C}_{\text {STIS. }}$. Long-term variations are best described with a fourth order polynomial function of time in Visit $A_{\text {STIS }}$, with the first-order term set to zero. In other visits the variations are best described with linear functions. Preliminary fits revealed an outlier in Visit $\mathrm{B}_{\text {STIS }}$ (caused by a spike in the stellar $\mathrm{H} \alpha$ line) and six outliers in Visit $\mathrm{C}_{\text {STIS }}$ (likely caused by the transit of a stellar spot), which were excluded from further analysis.

In a second step, we fitted the three visits together using a model that combines the polynomial variations with a transit light curve (calculated with the EXOFAST routines, Mandel \& Agol 2002; Eastman et al. 2013). The start and end times of the exposures were converted into $\mathrm{BJD}_{\mathrm{TDB}}$ from the $\mathrm{HJD}_{\mathrm{UTC}}$ times
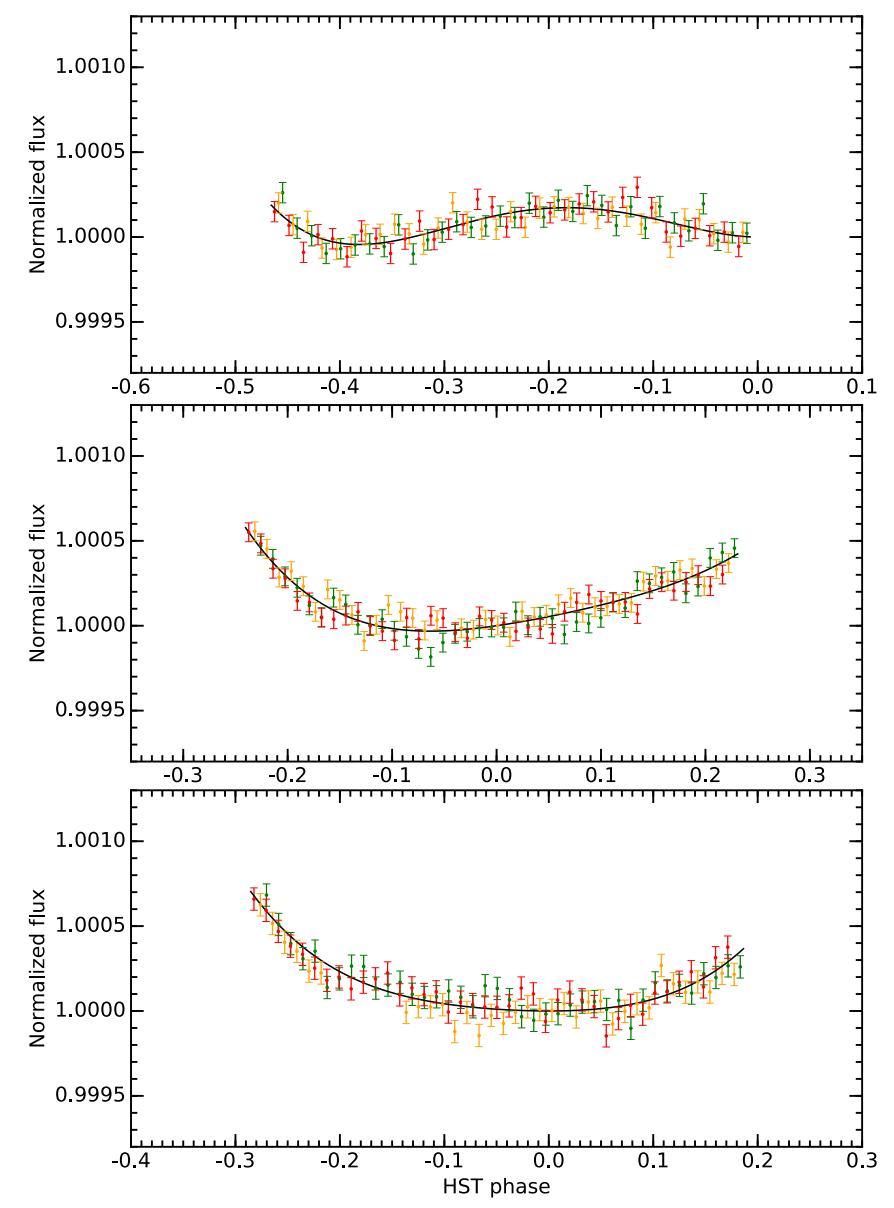

Fig. 8. Visible flux of $55 \mathrm{Cnc}$ phase-folded on the HST orbital period. Fluxes have been corrected for the long-term variations and transit light curve, to highlight the breathing variations (best-fitted with the solid black line). Color code is the same as in Fig. 7.

obtained from the file headers (Eastman et al. 2010). The model was oversampled in time and averaged within the time window of each exposure before comparison. The parameters of the model are the eight coefficients of the long-term polynomial variations, the ten coefficients of the breathing variations, the planet-tostar radii ratio $R_{\mathrm{p}} / R_{*}$, the mid-transit time at the epoch of our observations $T_{0}^{\mathrm{STIS}}$, the orbital inclination $i_{\mathrm{p}}$, and the quadratic limb-darkening coefficients $u_{1}$ and $u_{2}$. We fixed other system properties to the values given in Table 1 .

A preliminary fit was obtained with a Levenberg-Marquardt least-squares algorithm. We binned the residuals from this fit within nonoverlapping windows containing $N$ exposures, and measured the standard deviation $\sigma(N)$ of the binned residuals for increasing values of $N$ (e.g., Pont et al. 2006; Winn et al. 2009; Wilson et al. 2015). This revealed that $\sigma(N)$ decreases in $1 / \operatorname{sqrt}(N)$, which implies that there is no significant correlated noise (see Fig. 9). As a result, we set the uncertainties on the datapoints to the dispersion measured in the residuals (about $60 \mathrm{ppm}$ in each visit). We then sampled the posterior distributions of the model parameters using the Markov chain Monte Carlo (MCMC) Python software package emcee (Foreman-Mackey et al. 2013). Model parameters were used as jump parameters, replacing the inclination by its cosine, and the limbdarkening coefficients by the linear combinations $c_{1}=2 u_{1}+$ $u_{2}$ and $c_{1}=u_{1}-2 u_{2}$ (Holman et al. 2006). Uniform priors were used with the polynomial coefficients and $R_{\mathrm{p}} / R_{*}$. We imposed 
Table 4. Final values for the properties of $55 \mathrm{Cnc}$.

\begin{tabular}{lccl}
\hline \hline Parameter & Symbol & Value & Unit \\
\hline Planet-to-star radii ratio & $R_{\mathrm{p}} / R_{\star}$ & $0.0182 \pm 2 \times 10^{-4}$ & \\
Radius & $R_{\mathrm{p}}$ & $1.875 \pm 0.029$ & $R_{\text {Earth }}$ \\
Mass $^{\dagger}$ & $M_{\mathrm{p}}$ & $7.99_{-0.33}^{+0.32}$ & $M_{\text {Earth }}$ \\
Density $^{\text {Transit epoch }}$ & $\rho_{\mathrm{p}}$ & $6.66_{-0.40}^{+0.43}$ & $\mathrm{~g} \mathrm{~cm}^{-3}$ \\
Orbital period $^{\dagger}$ & $T_{0}^{\mathrm{STIS}}-2450000$ & $7063.2096_{-0.0004}^{+0.0006}$ & $\mathrm{BJD}_{\mathrm{TDB}}$ \\
Orbital inclination & $P$ & $0.7365474_{-1.410^{-6}}^{+1.310^{-6}}$ & $\mathrm{days}$ \\
Impact parameter $^{\text {Eccentricity }}{ }^{\dagger}$ & $i_{\mathrm{p}}$ & $83.59_{-0.44}^{+0.47}$ & $\mathrm{deg}$ \\
Argument of periastron $^{\dagger}$ & $b$ & $0.39 \pm 0.03$ & \\
Scaled semi-major axis $^{\dagger}$ & $e$ & $0.05 \pm 0.03$ & \\
Semi-major axis $^{\dagger}$ & $\omega$ & $86.0_{-33.4}^{+30.7}$ & $\mathrm{deg}$ \\
\hline
\end{tabular}

Notes: All values are derived from the HST/STIS transit analysis, except for parameters with a dagger derived from the velocimetry analysis, and reported from Table 3. Bulk density is derived from the posterior distributions on the planet mass and radius.

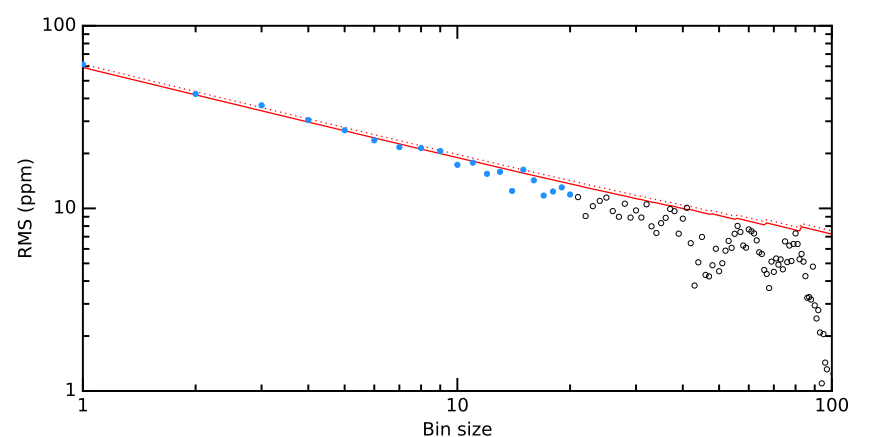

Fig. 9. RMS of binned residuals between the combined STIS visits and their best-fit model, as a function of bin size. The solid red line shows the best-fit noise model (quadratic combination of a Gaussian "white" noise and a constant correlated "red" noise), which was fitted over the blue measurements. The dotted red line shows a pure Gaussian noise model scaled to the RMS over individual exposures.

Gaussian priors on $\cos \left(i_{\mathrm{p}}\right)$, using the value from Demory et al. (2016a). We set a Gaussian prior on $T_{0}^{\text {STIS }}$ using the value derived from the velocimetry analysis (Sect. 3 ), and propagating the uncertainties on $P$ and $T_{0}$ onto the time of transit at the epoch of our observations. Quadratic limb-darkening coefficients were estimated in the SDSS $r$ ' band (centered at $612.2 \mathrm{~nm}$ with a width of $115 \mathrm{~nm}$ ) using the EXOFAST calculator ${ }^{2}$ (Eastman et al. 2013) and the stellar temperature, gravity, and metallicity from Table 1. We varied these parameters within their $1 \sigma$ uncertainties to determine uncertainties on $u_{1}$ and $u_{2}$ (found to be dominated by the error on the metallicity). The mean and errors so derived $\left(u_{1}=0.545 \pm 0.008\right.$ and $\left.u_{2}=0.186 \pm 0.004\right)$ were used as Gaussian priors in the MCMC. We initialized 300 walkers that were started at random points in the parameter space, close to the preliminary fit. We ran the walkers for 7000 steps and removed a conservative 3000 steps as burn-in. We checked that all walkers converged to the same solution, before thinning their chains using the maximum correlation length of all parameters. The final thinned and merged chain contains about 4000 independent samples. We set the best-fit values for the model parameters to

2 http://astroutils.astronomy.ohio-state.edu/exofast/ limbdark. shtml the medians of the posterior probability distributions and evaluated their $1 \sigma$ uncertainties by taking limits at $34.15 \%$ on either side of the median. Results are given in Table 4 . The best-fit transit light curve is shown in Fig. 10. Taking into account the uncertainty on the stellar radius, the corresponding planet-to-star radius ratio $R_{\mathrm{p}} / R_{\star}=0.0182 \pm 0.0002$ corresponds to an optical radius $R_{\mathrm{p}}=1.875 \pm 0.029 R_{\text {Earth }}$. We combined the posterior probability distributions obtained for the mass and radius of the planet to obtain the distribution for the density, and derived $\rho_{\mathrm{p}}=$ $6.66_{-0.40}^{+0.43} \mathrm{~g} \mathrm{~cm}^{-3}$.

\subsection{Analysis of APT transit light curve}

The transit of $55 \mathrm{Cnce}$ was detected from the ground by de Mooij et al. (2014), using differential photometry obtained with ALFOSC on the 2.5-m Nordic Optical Telescope. They measured a transit depth of $0.0198_{-0.0014}^{+0.0013}$ in the Johnson BVR bands, which is consistent with our STIS measurement. We searched for the transit in our normalized APT differential photometry (Sect. 2.2) using the EXOFAST model described in Sect. 4.2. In a first step, we fitted the transit depth, transit epoch, and orbital period and fixed all other properties to the values given in Tables 1 and 4. The average Strömgren $b$ and y passbands (centered at 467 and $547 \AA$, respectively) overlap with the STIS spectral range, and we consider it reasonable to use the limb-darkening parameters derived in Sect. 4.2 given the precision of the APT data. Errors on datapoint were set to the dispersion of the residuals from a preliminary best-fit. We found the transit at a period $P=0.736547 \pm 2 \times 10^{-6}$ days and epoch $T_{0}^{\mathrm{APT}}=2457063.201 \pm 0.007 \mathrm{BJD}_{\mathrm{TDB}}$, in good agreement with the results from space-borne photometry (Table 4). In a second step, we thus fitted the transit depth alone (Fig. 11), all other properties being fixed to their values in Table 4 . We obtain $R_{\mathrm{p}} / R_{*}=0.0228 \pm 0.0023$, which is marginally larger $(2 \sigma)$ than the STIS value derived in Sect. 4.2.

\section{Interior characterization of 55 Cnce}

Successive measurements of the mass and radius of $55 \mathrm{Cnc}$ e have been used to constrain its interior composition, ranging from a planet with a high-mean-molecular-weight atmosphere 
Table 5. Interior parameters and corresponding prior ranges.

\begin{tabular}{lllll}
\hline \hline Layer & Parameter & Symbol & Prior range & Distribution \\
\hline $\begin{array}{l}\text { Core } \\
\text { Mantle }\end{array}$ & Radius & $r_{\text {core }}$ & $(0.01-1) r_{\text {core+mantle }}$ & Uniform in $r_{\text {core }}^{3}$ \\
& Composition & $\mathrm{Fe} / \mathrm{Si}_{\text {mantle }}$ & $0-\mathrm{Fe} / \mathrm{Si}_{\text {star }}$ & Uniform \\
& Radius of rocky interior & $r_{\text {core+mantle }} / \mathrm{Si}_{\text {mantle }}$ & $\mathrm{Mg} / \mathrm{Si}_{\text {star }}$ & Gaussian \\
Nongaseous water layer & Mass fraction & $m_{\text {water }}($ wet case $)$ & $(0-0.9) M_{p}$ & Uniform in $r_{\text {core+mantle }}^{3}$ \\
& & $m_{\text {water }}($ dry case $)$ & 0 & Uniform \\
Gas layer & Bottom pressure & $P_{\text {batm }}$ & $P_{\text {out }}-P_{\text {batm,max }}$ & ln-uniform \\
& Temperature coefficient & $\alpha$ & $0.5-1$ & Uniform \\
& Mean molecular weight & $\mu$ & $2.3-50\left(\mathrm{~g} \mathrm{~mol}^{-1}\right)$ & Uniform \\
\hline
\end{tabular}

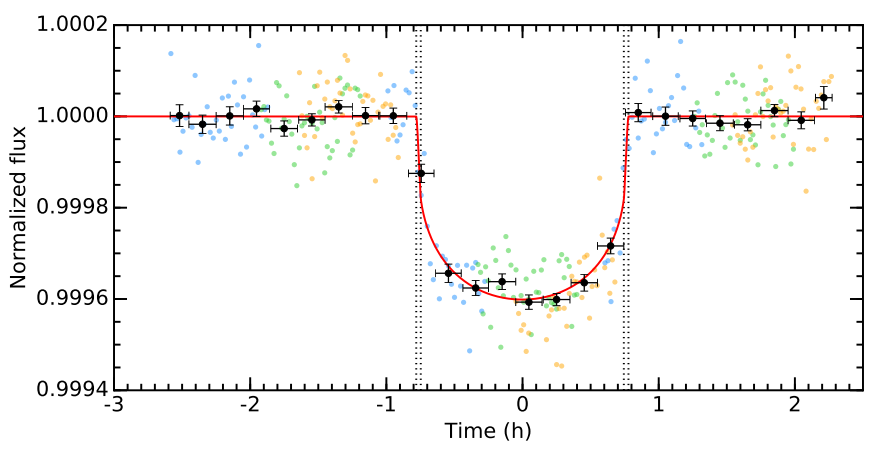

Fig. 10. STIS transit light curve of $55 \mathrm{Cnc}$ e in the visible band. Fluxes have been corrected for the breathing and long-term variations in Visit $\mathrm{A}_{\text {STIS }}$ (blue), $\mathrm{B}_{\text {STIS }}$ (green), and $\mathrm{C}_{\text {STIS }}$ (orange). Black points show binned exposures. The red line is the best-fit transit light curve.

(Demory et al. 2011) to a planet with no atmosphere and a silicate-rich (Winn et al. 2011) or carbon-rich (Madhusudhan et al. 2012) interior. Our new estimates of planetary radius and mass (Table 4) are consistent with previous measurements by Nelson et al. (2014) and Demory et al. (2016a; $R_{\mathrm{p}}=1.91 \pm$ $\left.0.08 R_{\text {Earth }} ; M_{\mathrm{p}}=8.08 \pm 0.31 M_{\text {Earth }}\right)$, and their improved precision allow us carry further the interior characterization of $55 \mathrm{Cnce}$. We used the generalized Bayesian inference analysis of Dorn et al. (2017b) to rigorously quantify interior degeneracy. We investigated two different scenarios: a dry interior that is comprised of gas and rock only, and a wet scenario in which a nongaseous water layer is present underneath the gas layer.

The data that we considered as input to the interior characterization are planet mass, planet radius, irradiation from the host star (i.e., semi-major axes $a=0.01544$ au, stellar effective temperature $T_{\text {star }}=0.895 T_{\odot}$, and stellar radius $R_{\text {star }}=0.943 R_{\odot}$ ), as well as the stellar abundances. The stellar abundances are used as a proxy for the bulk composition of the planet. We followed the compilation of Dorn et al. (2017a), where the derived median values are $\mathrm{Fe} / \mathrm{Si}_{\text {bulk }}=1.86 \pm 1.49, \mathrm{Mg} / \mathrm{Si}_{\text {bulk }}=0.93 \pm 0.77$, $m_{\mathrm{CaO}}=0.013 \mathrm{wt} \%, m_{\mathrm{Al}_{2} \mathrm{O}_{3}}=0.062 \mathrm{wt} \%, m_{\mathrm{Na}_{2} \mathrm{O}}=0.024 \mathrm{wt} \%$. We note that the uncertainties on the abundance constraints are high $(\sim 80 \%)$.

\subsection{Method}

The compositional and structural interior parameters that we aim to quantify given the data are given in Table 5. In the dry scenario, the planetary interior is assumed to be composed of a pure iron core, a silicate mantle comprising major and minor rock-forming oxides $\mathrm{Na}_{2} \mathrm{O}-\mathrm{CaO}-\mathrm{FeO}-\mathrm{MgO}-\mathrm{Al}_{2} \mathrm{O}_{3}-\mathrm{SiO}_{2}$, and a gas layer. In the wet scenario, the mantle is topped by a

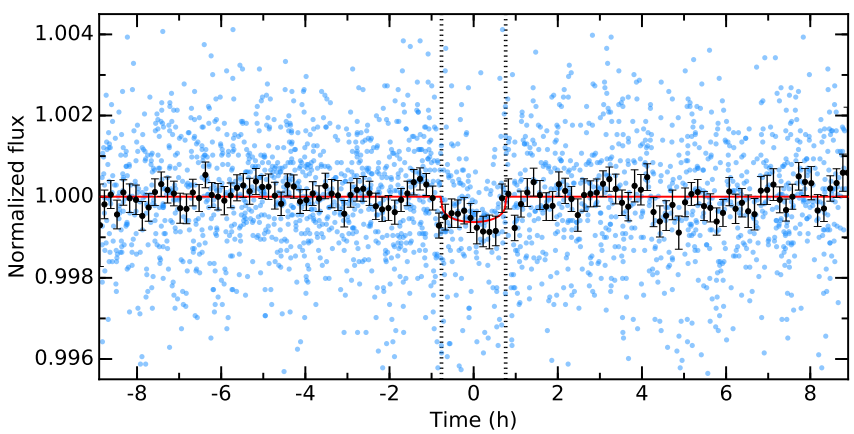

Fig. 11. APT transit light curve of $55 \mathrm{Cnce}$ in the Strömgren b and $\mathrm{y}$ bands. Black points show binned exposures. The red line is the best-fit transit light curve.

nongaseous water layer, which can be high-pressure ice and/or superionic water given the high temperature of $55 \mathrm{Cnc}$ e (see also Dorn et al. 2017a). A gas layer on top of the water layer is still necessary to impose a pressure high enough to prevent evaporation of water. In both the dry and wet scenarios, the gas layer is assumed to be dominated by gas of mean molecular weights that range from hydrogen to heavy compounds (e.g., $\mathrm{N}_{2}, \mathrm{H}_{2} \mathrm{O}, \mathrm{CO}_{2}$, $\left.\mathrm{CO}, \mathrm{O}_{2}, \mathrm{Na}+, \mathrm{Ca}^{2+}\right)$, i.e., $2.3<\mu<50\left(\mathrm{~g} \mathrm{~mol}^{-1}\right)$.

The prior distributions of the interior parameters are listed in Table 5. The priors were chosen conservatively. The cubic uniform priors on $r_{\text {core }}$ and $r_{\text {core+mantle }}$ reflect equal weighing of masses for both core and mantle. Prior bounds on $\mathrm{Fe} / \mathrm{Si}_{\text {mantle }}$ and $\mathrm{Mg} / \mathrm{Si}_{\text {mantle }}$ are determined by the host star's photospheric abundance proxies, whenever abundance constraints are considered. Since iron is distributed between core and mantle, $\mathrm{Fe} / \mathrm{Si}_{\text {bulk }}$ only sets an upper bound on $\mathrm{Fe} / \mathrm{Si}_{\text {mantle }}$. For the gas layer the maximum surface pressure $\left(P_{\text {batm }}\right)$ is determined by the maximum gas mass that a super-Earth can accrete and retain (Ginzburg et al. 2016).

The structural model for the interior uses self-consistent thermodynamics for core, mantle, and water layer. For the core density profile, we use the equation of state (EoS) fit of iron in the hcp (hexagonal close-packed) structure provided by Bouchet et al. (2013) on ab initio molecular dynamics simulations. For the silicate mantle, we computed equilibrium mineralogy and density as a function of pressure, temperature, and bulk composition by minimizing Gibbs free energy (Connolly 2009). We assumed an adiabatic temperature profile within core and mantle.

For the nongaseous water layer, we followed Vazan et al. (2013) using a quotidian equation of state (QEOS) and above a pressure of $44.3 \mathrm{GPa}$, we use the tabulated EoS from Seager et al. (2007). An adiabatic temperature profile is also assumed for the water layer. If a water layer is present, there must be a gas 


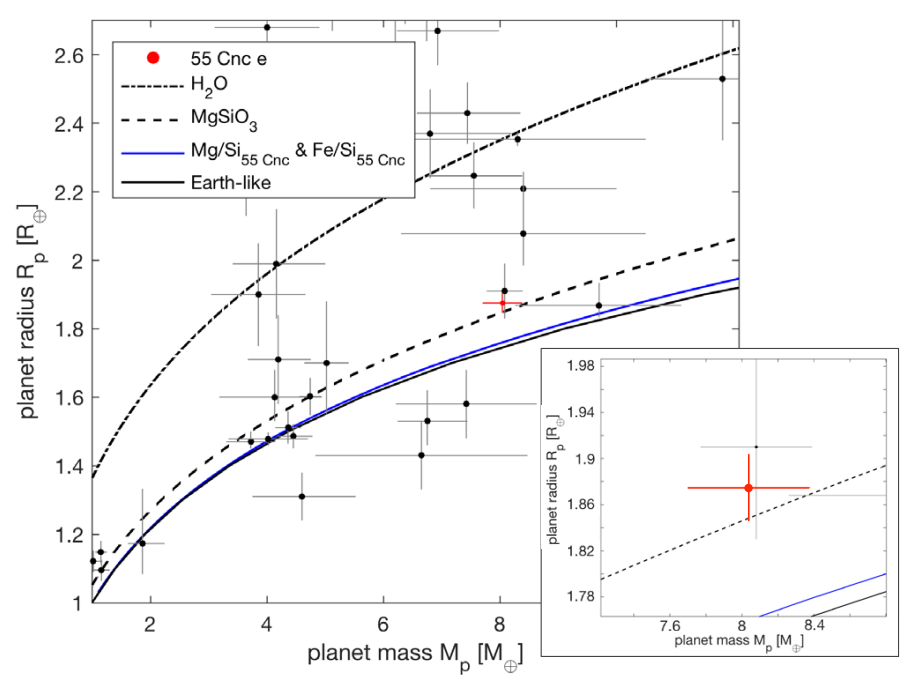

Fig. 12. Mass and radius of $55 \mathrm{Cnc}$ e (shown in red) in comparison with four mass-radius-relationships of idealized rocky interiors: a pure water composition, the least-dense purely - silicate interior represented by $\mathrm{MgSiO}_{3}$, an interior of an iron core and a iron-free mantle that fits the stellar refractory abundances of $55 \mathrm{Cnc}$ (mass ratios: $\mathrm{Mg} / \mathrm{Si}=0.927$ and $\mathrm{Fe} / \mathrm{Si}=1.855$ ), and an Earth-like composition. We show exoplanets with mass known to better than $30 \%$ (error bars represent $1-\sigma$ uncertainties on their mass and radius).

layer on top that imposes a pressure that is at least as high as the vapor pressure of water.

For the gas layer, we used a simplified atmospheric model for a thin, isothermal atmosphere in hydrostatic equilibrium and ideal gas behavior, which is calculated using the scale-height model. The model parameters that parameterize the gas layer and that we aim to constrain are the pressure at the bottom of the gas layer $P_{\text {batm }}$, the mean molecular weight $\mu$, and the mean temperature (parameterized by $\alpha$, see below). The thickness of the opaque gas layer $d_{\text {atm }}$ is given by

$d_{\mathrm{atm}}=H \ln \frac{P_{\mathrm{batm}}}{P_{\text {out }}}$,

where the amounts of opaque scale-heights $H$ is determined by the ratio of $P_{\text {batm }}$ and $P_{\text {out }} . P_{\text {out }}$ is the pressure level at the optical photosphere for a transit geometry that we fix to $20 \mathrm{mbar}$ (Fortney 2007). The scale-height $H$ is the increase in altitude for which the pressure drops by a factor of $e$ and can be expressed by

$H=\frac{T_{\mathrm{atm}} R^{*}}{g_{\mathrm{batm}} \mu}$,

where $g_{\text {batm }}$ and $T_{\text {atm }}$ are gravity at the bottom of the atmosphere and mean atmospheric temperature, respectively. $R^{*}$ is the universal gas constant $\left(8.3144598 \mathrm{~J} \mathrm{~mol}^{-1} \mathrm{~K}^{-1}\right)$ and $\mu$ the mean molecular weight. The mass of the atmosphere $m_{\text {atm }}$ is directly related to the pressure $P_{\text {batm }}$ as

$m_{\mathrm{atm}}=4 \pi P_{\mathrm{batm}} \frac{r_{\mathrm{batm}}^{2}}{g_{\mathrm{batm}}}$,

where $r_{\text {batm }}$ is the radius at the bottom of the atmosphere. The atmosphere's constant temperature is defined as

$T_{\text {atm }}=\alpha T_{\text {star }} \sqrt{\frac{R_{\text {star }}}{2 a}}$,
Table 6. Interior parameter estimates for dry and wet scenario

\begin{tabular}{lll}
\hline \hline Interior parameter & Wet & Dry \\
\hline $\log _{10}\left(P_{\text {batm }}[\mathrm{atm}]\right)$ & $4.18_{-1.17}^{+1.11}$ & $2.76_{-2.14}^{+1.77}$ \\
$\mu\left(\mathrm{g} \mathrm{mol}^{-1}\right)$ & $28.85_{-13.40}^{+12.98}$ & $13.49_{-7.72}^{+17.85}$ \\
$\alpha$ & $0.74_{-0.15}^{+0.16}$ & $0.78_{-0.16}^{+0.14}$ \\
$r_{\text {gas }} / R_{p}$ & $0.02_{-0.01}^{+0.02}$ & $0.03_{-0.02}^{+0.04}$ \\
$m_{\text {water }} / M_{p}$ & $0.08_{-0.04}^{+0.06}$ & - \\
$r_{\text {core }+ \text { mantle }} / R_{p}$ & $0.87_{-0.06}^{+0.05}$ & $0.97_{-0.04}^{+0.02}$ \\
$r_{\text {core }} / r_{\text {core+mantle }}$ & $0.40_{-0.11}^{+0.10}$ & $0.31_{-0.09}^{+0.09}$ \\
$\mathrm{Fe} / \mathrm{Si}_{\text {mantle }}$ & $1.13_{-0.75}^{+1.22}$ & $0.69_{-0.46}^{+0.90}$ \\
$\mathrm{Mg} / \mathrm{Si}_{\text {mantle }}$ & $1.01_{-0.55}^{+0.65}$ & $1.25_{-0.62}^{+0.66}$ \\
\hline
\end{tabular}

Notes. One- $\sigma$ uncertainties of the one-dimensional marginalized posteriors are listed.

where $R_{\text {star }}$ and $T_{\text {star }}$ are radius and effective temperature of the host star and $a$ the planet semi-major axis. The factor $\alpha$ accounts for possible cooling of the atmosphere and can vary between 0.5 and 1, which is equivalent to observed ranges of albedos among solar system bodies ( 0.05 for asteroids up to 0.96 for Eris). Significant warming in the thin gas layers is neglected, which can result in an underestimation of gas layer thicknesses with consequences for the predicted interior parameters for water and rock layers.

Here, we calculate globally - averaged interior profiles that do not account for hemispheric variations. However, we note that 55 Cnce shows temperature variations between day- and nightside estimated to be around 1300-3000 K (Demory et al. 2016a,b). A plausible explanation is the presence of a molten magma ocean on the dayside that interacts with the gas envelope above (Elkins-Tanton 2012), while the rock surface on the nightside of the tidally - locked planet could be solidified. Furthermore, the possibility for variable features in the exosphere and thermal emission of $55 \mathrm{Cnce}$ (Ridden-Harper et al. 2016; Demory et al. 2016a) call for more complex models that would integrate the dynamic interaction between interior and atmosphere as well as the interaction between planet and star. This, however, is beyond the scope of our study. We refer the reader to model II in Dorn et al. (2017b) for more details on both the inference analysis and the structural model.

\subsection{Results}

Using the generalized MCMC method, we obtain a large number of models $\left(\sim 10^{6}\right)$ that sample the posterior distribution of possible interiors. For the interior parameters of interest, we obtain posterior distributions that are plotted in Figs. 13 and 14. Parameter estimates are summarized in Table 6.

In the dry scenario (Fig. 13), we find interiors that are dominated by a solid interior with radius fractions of $r_{\text {core }+ \text { mantle }}=$ $0.97_{-0.04}^{+0.02} R_{\mathrm{p}}$. The corresponding radius of the gas envelope $\left(0.03_{-0.02}^{+0.04} R_{\mathrm{p}}\right.$ is consistent with an independent analysis of $55 \mathrm{Cnc}$ e composition performed by Crida et al. (2018). We note that in the dry scenario the data allows for the complete absence of a gas envelope, but this possibility has to be considered in light of other observations of the planet (Sect. 6.2). The individual parameters of the gas layer are poorly constrained, except for the thickness of the gas layer. 


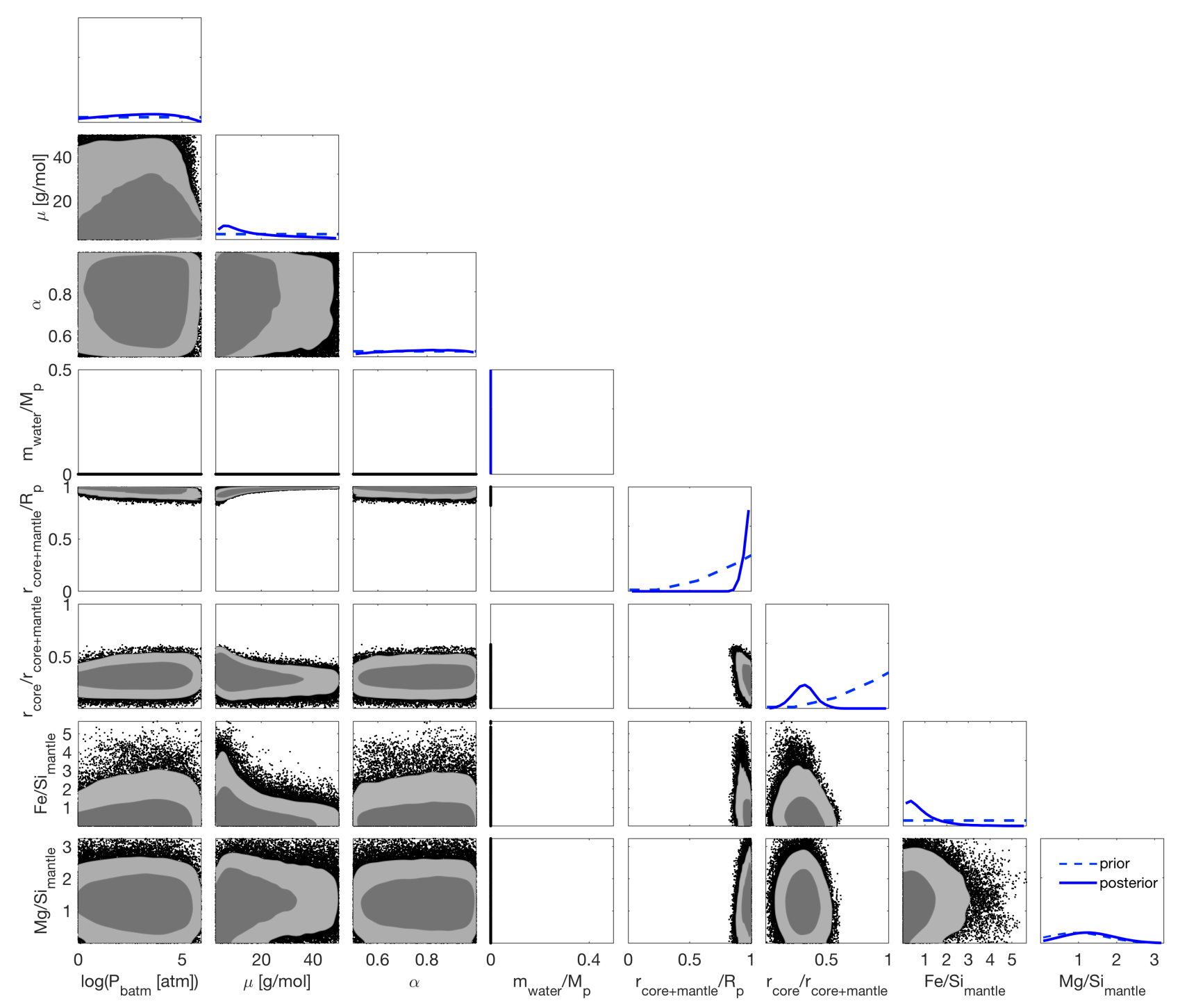

Fig. 13. Sampled one- and two-dimensional marginal posterior for interior parameters of the dry case: (panel $a)$ surface pressure $P_{\text {batm }},($ panel $b)$ mean molecular weight $\mu$, (panel c) $\alpha$, (panel $d$ ) water mass fraction $m_{\text {water }} / M_{\mathrm{p}}$, which is always zero in the dry case, (panel $e$ ) size of rocky interior $r_{\text {mantle+core }} / R_{\mathrm{p}}$, (panelf) relative core size $r_{\text {core }} / r_{\text {mantle+core }}$, (panel $g, h$ ) mantle composition in terms of Fe $/ \mathrm{Si}_{\text {mantle }}$ and $\mathrm{Mg} / \mathrm{Si}_{\text {mantle. }}$ The prior distributions are shown in dashed blue.

In the wet scenario, when we allowed for a nongaseous water layer underneath the gas layer, we estimate the possible water mass fraction to be $8_{-4}^{+6} \%$. This result is in agreement with the water mass fraction of $8 \pm 3 \%$ estimated by Lopez (2017). By adding a water layer, we imposed the condition that there must be a gas layer on top that imposes a pressure that is at least as high as the vapor pressure of water. This condition has a major influence on our estimates of gas mas fractions, in other words the gas layer has a minimum surface pressure of $200 \mathrm{~atm}$. In order to fit bulk density while keeping the gas mass high, low mean molecular weights are excluded. Thick gas and water layers require a smaller rocky interior $\left(r_{\text {core+mantle }}\right)$ in order to fit the total radius. At the same time the total mass can only be fit by a denser rocky interior, which is realized by a larger core size and an iron-enriched mantle while remaining within the bounds of the the abundance constraint $\mathrm{Fe} / \mathrm{Si}_{\text {bulk }}$.

The presence of a nongaseous water layer on $55 \mathrm{Cnce}$ requires a thinner gas envelope (2\% radius fraction) than in the dry scenario ( $3 \%$ radius fraction). For the wet scenario, there is a marginal preference of low-density interiors with denser rocky cores. In order to decide which scenario is more likely, we discuss in Sect. 6.2 our results in light of additional data, specifically those on exosphere observations.

\section{Discussion}

\subsection{Long-term variations in 55 Cnce radius}

As mentioned in Sect. 1, there is evidence for a variable source of opacity around $55 \mathrm{Cnce}$. This could trace, for example, temporal variability in an atmosphere subjected to exchange of matter with surface molten rocks as well as losses to space caused by stellar irradiation. This scenario could further be responsible for variations in the apparent radius of $55 \mathrm{Cnc}$ e over time (Demory et al. 2016b). To investigate this possibility, we first fitted the individual transit depth in each HST/STIS visit with the model described in Sect. 4.2, all other properties being fixed to their best-fit values (Table 4 ). We derived $R_{\mathrm{p}} / R_{*}=0.0186 \pm$ $0.0003,0.0177 \pm 0.0002$, and $0.0190 \pm 0.0003$ in visits $\mathrm{A}, \mathrm{B}$, and $\mathrm{C}$, respectively. We show in Fig. 15 those values as a function 

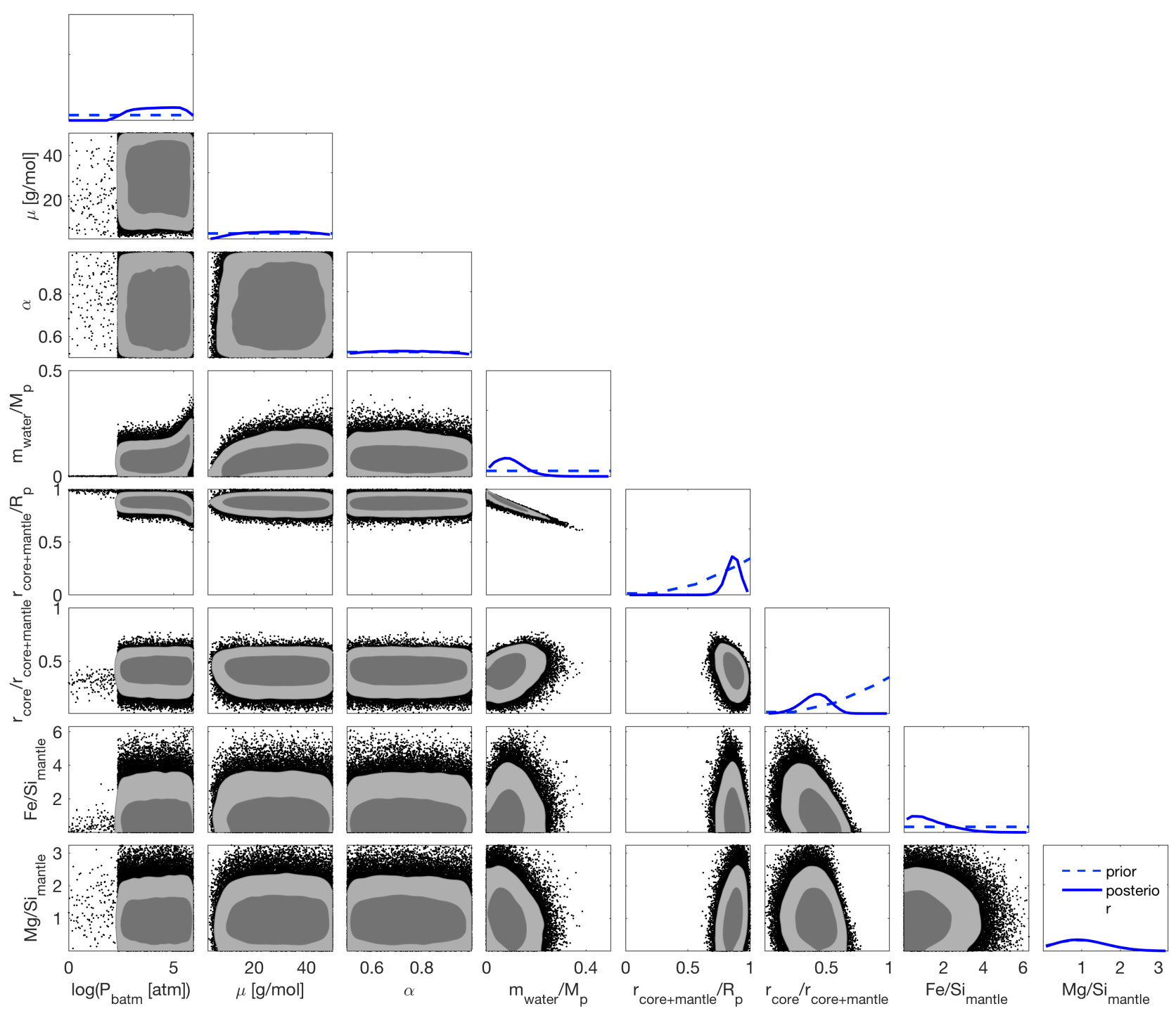

Fig. 14. Sampled one- and two-dimensional marginal posterior for interior parameters of the wet case: (panel $a)$ surface pressure $P_{\text {batm }},($ panel $b)$ mean molecular weight $\mu$, (panel c) $\alpha$, (panel $d$ ) water mass fraction $m_{\text {water }} / M_{\mathrm{p}}$, (panel $e$ ) size of rocky interior $r_{\text {mantle }+ \text { core }} / R_{\mathrm{p}}$, (panel $f$ ) relative core size $r_{\text {core }} / r_{\text {mantle+core }}$, (panel $g, h$ ) mantle composition in terms of $\mathrm{Fe} / \mathrm{Si}_{\text {mantle }}$ and $\mathrm{Mg} / \mathrm{Si}_{\text {mantle }}$. The prior distributions are shown in dashed blue.

of time, along with all measurements of 55 Cnc e planet-to-star radius ratio available in the litterature. Ground-based and spaceborne transit observations have been obtained between 2011 and 2017 in visible and infrared bands. Apart for marginally lower value in the second of six Spitzer measurements (Demory et al. 2016 b), early planet-to-star radius ratios of 55 Cnce measured with MOST (Gillon et al. 2012; Dragomir et al. 2014), Spitzer (Demory et al. 2011, 2016b,a; Gillon et al. 2012), and ALFOSC (de Mooij et al. 2014) are consistent within their uncertainties (Fig. 15). The five measurements obtained at a much higher precision with HST/STIS (this work) and WFC3 (Tsiaras et al. 2016) are consistent with these older values, and with the planet radius we derive from the common fit to the STIS data (within $2.4 \sigma$ ). There is thus no evidence for long-term variations in the apparent size of $55 \mathrm{Cnc}$ over timescales of a few years. In contrast, the planet-to-star radius ratio obtained in STIS Visit B is significantly lower $(\sim 4 \sigma)$ than the three most recent HST measurements, including the one from Visit $\mathrm{C}$ obtained only 12 days later. While we cannot exclude statistical variations, systematic linked to the incompleteness of the STIS individual transits, or stellar variability (although $55 \mathrm{Cnc}$ is a quiet star at optical wavelengths), the lower planet radius in Visit B might trace temporal variability in $55 \mathrm{Cnc}$ e properties over time-scales of a few days or weeks, as suggested by Demory et al. (2016b).

\subsection{Disentangling between a dry and wet 55 Cnce}

For the interior characterization, we have used two scenarios (dry or wet) that differ in their prior assumptions on the presence of a water layer. In either scenarios, we find that a gas fraction likely contributes to the radius by few percents, which can also be inferred from bulk density (Fig. 12). However, we find that our interior estimates of the rocky and volatile-rich compounds strongly depend on the scenario. Two implications can be made from this. First, besides the available data, a priori assumptions can contain crucial information on interiors. Second, it is difficult from the measured mass and radius alone to decide whether the wet or the dry case are more likely, which underlines the importance of atmospheric characterization to determine the nature of exoplanets. Our characterization of the interior is 


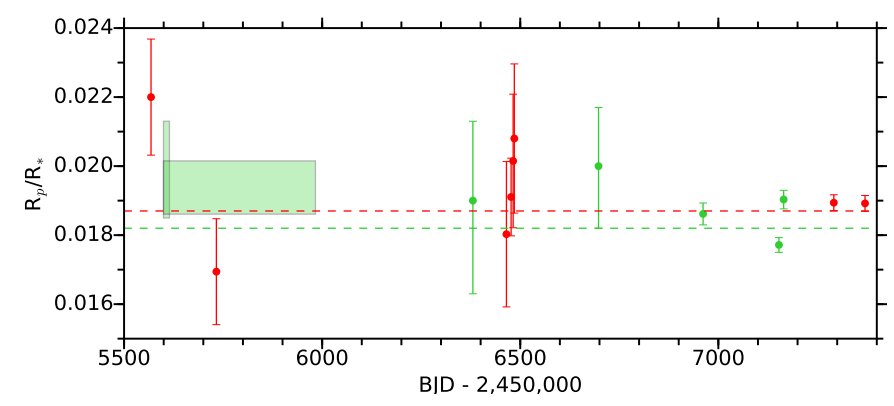

Fig. 15. Measurements of $55 \mathrm{Cnc}$ e planet-to-star radius ratio over time. Green points were obtained in optical bands with MOST (Gillon et al. 2012; Dragomir et al. 2014), ALFOSC (de Mooij et al. 2014), HST/STIS (this paper). The first two values are represented as rectangles because they were derived over extended periods of time. The dashed green line shows the value obtained from the fit to the three combined STIS visits. Red points were obtained in infrared bands with Spitzer (Demory et al. 2016b) and HST/WFC3 (Tsiaras et al. 2016). The dashed red line shows the value obtained from the fit to the combined Spitzer visits (Demory et al. 2016b,a).

solving a static problem and does not account for the evolution of the planet. Over the planet's lifetime, the intense irradiation from the star can lead to significant mass loss from the planetary atmosphere. We plot in Fig. 16, the atmospheric mass-loss rate from $55 \mathrm{Cnc}$ e for different mean molecular weights $\mu$, assuming an energy-limited regime (Lecavelier des Etangs 2007; Erkaev et al. 2007) with two representative evaporation efficiencies $\eta$ (0.01 and 0.2; Salz et al. 2016). The mass loss is described as

$\dot{M}=\frac{\pi \eta F_{\mathrm{XUV}} R_{\text {base }}^{2}}{E_{g}}$,

where $F_{\mathrm{XUV}}$ is the XUV flux at the planets age and orbital distance to the star (taken from Bourrier et al. 2018), $E_{g}$ is the gravitational potential at $R_{\text {base }}$. $R_{\text {base }}$ is the planet radius at the XUV photosphere

$R_{\text {base }} \approx R_{\mathrm{p}}+H \ln \left(\frac{P_{\text {photo }}}{P_{\text {base }}}\right)$,

where $P_{\text {photo }}$ and $P_{\text {base }}$ are set to typical values of 20 mbar and 1 nbar, respectively (Lopez 2017). The dependency of the mass loss rate on $\mu$ is due to the scale height $H$, which is the scale height in the regime between the optical and the XUV photosphere

$H=\frac{T_{\mathrm{eq}} R^{*}}{g_{\mathrm{surf}} \mu}$,

where $g_{\text {surf }}$ is surface gravity and $R^{*}$ is the universal gas constant $\left(8.3144598 \mathrm{~J} \mathrm{~mol}^{-1} \mathrm{~K}^{-1}\right)$. It is clear that a primordial hydrogen layer would have been lost within a tiny fraction of the planets lifetime $(\sim 80000 \mathrm{yr})$, which is consistent with the high mean molecular weights that we derived in the dry $\left(\mu=13.5_{-7.7}^{+17.9} \mathrm{~g} \mathrm{~mol}^{-1}\right)$ and wet $\left(\mu=28.9_{-13.4}^{+13.0} \mathrm{~g} \mathrm{~mol}^{-1}\right)$ scenarios. As a natural result of the strongly irradiated water layer in the wet scenario, we would expect the gas layer to be dominated by steam. The planet mass and radius favors a heavier atmosphere in this scenario, and indeed there is no observational evidence for an extended water envelope (Esteves et al. 2017).

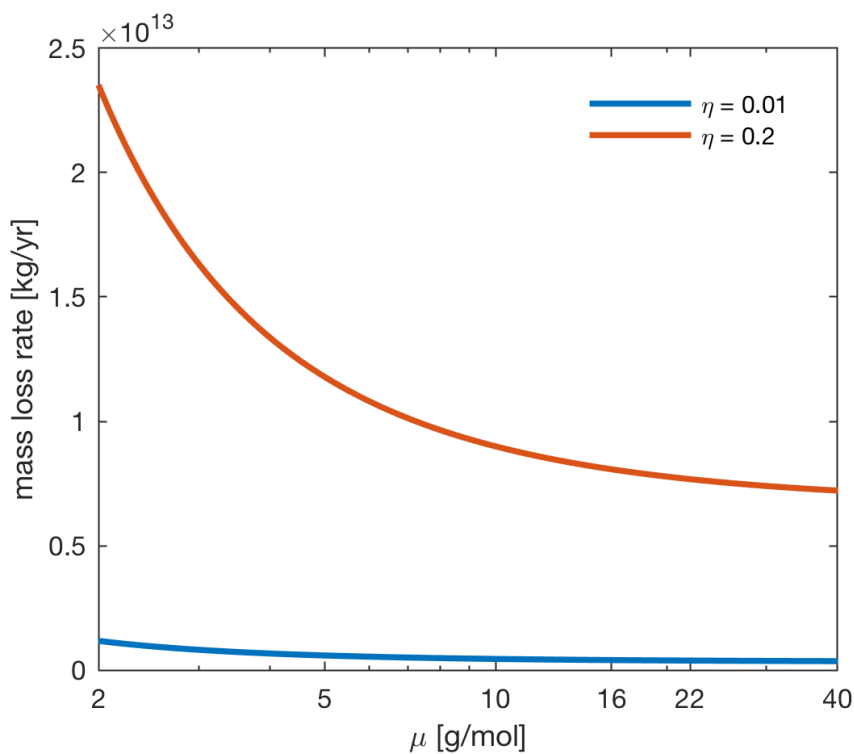

Fig. 16. Mass-loss rate from $55 \mathrm{Cnc}$ e in the energy-limited regime (for a conservative range of efficiencies), as a function of the atmospheric mean molecular weight.

Furthermore, steam would get photodissociated at high-altitudes and sustain an upper atmosphere of hydrogen, which has not been detected (Ehrenreich et al. 2012). Finally, Fig. 16 suggests that this hydrogen envelope would be lost quickly, depleting the steam envelope and underlying water layer. These additional constraints thus favor the dry scenario over the wet scenario.

In the dry case we find a gas radius fraction of $r_{\text {gas }} / R_{\mathrm{p}}=3_{-2}^{+4} \%$, which is compatible within $2 \sigma$ with an atmosphere-less body. However, infrared photometric data support the existence of an atmosphere of heavy weight molecules and inefficient heat-redistribution (Demory et al. 2016a; Angelo \& Hu 2017). 55 Cnc e could be surrounded by an atmosphere dominated by rock-forming elements, continually replenished by vaporization of, for example, silicates on top of a possible magma ocean. The existence of such a mineral-rich and water-depleted atmosphere was predicted for hot rocky super-Earths by Ito et al. (2015), assuming that gas and melted rocks of the magma ocean are in equilibrium. A search for atmospheric escape at FUV wavelengths revealed strong variations in the lines of the star, possibly arising from interactions between $55 \mathrm{Cnc}$ e and its star, but no clear signature of a metalrich exosphere (Bourrier et al. 2018). Nonetheless, a mineral atmosphere could explain the inefficient heat-redistribution and relatively high night-side temperature of the planet (Demory et al. 2016a; Zhang \& Showman 2017), and would be a likely origin for the sodium and ionic calcium possibly detected in the exosphere of $55 \mathrm{Cnc}$ e (Ridden-Harper et al. 2016). The mean molecular weight of our simple model is consistent with the presence of heavy species such as calcium $\left(\mu_{\mathrm{Ca}}=40.1 \mathrm{~g} \mathrm{~mol}^{-1}\right)$, sodium $\left(\mu_{\mathrm{Na}}=22.99 \mathrm{~g} \mathrm{~mol}^{-1}\right)$, or oxygen $\left(\mu_{\mathrm{O}}=16 \mathrm{~g} \mathrm{~mol}^{-1}\right)$. However, a gas layer dominated by one of these species, and with a radius fraction of $0.03 R_{p}$, would have surface pressures of 3700 and 90 bar, respectively (at $\alpha=1$ ). Such high surface pressures are at odds with the maximum surface pressure of $\sim 0.1$ bar estimated by Ito et al. (2015) for a mineral atmosphere in equilibrium with molten rock. The uncertainties we derive for the gas layer thicknesses and mean molecular weights do not 
allow us to distinguish between a mineral atmosphere and a gas layer dominated by molecules such as $\mathrm{CO}$ or $\mathrm{N}_{2}$, as suggested by the infrared phase curve of $55 \mathrm{Cnc}$ (Angelo \& Hu 2017; Hammond \& Pierrehumbert 2017).

\section{Conclusion}

We analyzed the long-term activity of $55 \mathrm{Cnc}$ using two decades of photometry and spectroscopy data. A solar-like cycle $\left(P_{\text {mag }}=10.5 \mathrm{yr}\right)$ is detected in all datasets, along with the stellar rotational modulation ( $P_{*}=38.8$ days $)$, confirming that $55 \mathrm{Cnc}$ is an old $(\sim 10 \mathrm{Gyr})$ and quiet star. The magnetic cycle was included for the first time in the velocimetric analysis of the system, allowing us to update the orbital and mass properties of the five known planets. Our results are consistent with past publications, except for significant differences in the period of the outermost planet. This is likely because its period is on the same order as that of the magnetic cycle, which we also detect in the radial velocity data.

The innermost planet $55 \mathrm{Cnc} \mathrm{e}$ is one of the most massive known USP planets, an iconic super-Earth that is a target of choice to understand the formation and evolution of small close-in planets. It orbits one of the brightest exoplanet hoststars, which allowed us to detect its transit in APT ground-based differential photometry. However, despite extensive observations across the entire spectrum, the nature of $55 \mathrm{Cnc}$ e remains shrouded in mistery. A precise knowledge of the planet density is necessary to determine its composition and structure, and we combined our derived planet mass $\left(M_{\mathrm{p}}=8.0 \pm 0.3 M_{\text {Earth }}\right)$ with refined measurement of its optical radius derived from HST/STIS observations $\left(R_{\mathrm{p}}=1.88 \pm 0.03 R_{\text {Earth }}\right.$ over 530 $750 \mathrm{~nm})$ to revise the density of 55 Cnc e $\left(\rho=6.7 \pm 0.4 \mathrm{~g} \mathrm{~cm}^{-3}\right)$. This result suggests that the super-Earth is too light to be purely made of silicate (Fig. 12), and we thus modeled its interior structure by allowing for water and gas layers. The precision on the mass and radius of $55 \mathrm{Cnc}$ does not allow us to conclude on the existence of a non-gaseous water layer, but the orbit of the planet at the fringes of the stellar corona $\left(a_{\mathrm{p}}=3.5 R_{*}\right)$, the nondetection of an extended hydrogen and water atmosphere, the possible detection of exospheric sodium and calcium, and the infrared mapping of the planet, all strongly point toward the absence of a significant water layer. Regarding the gas layer, the bulk density of $55 \mathrm{Cnc}$ e clearly excludes the presence of a $\mathrm{H} / \mathrm{He}$ envelope, despite the recent claim by Tsiaras et al. (2016). Even small amounts of hydrogen would drastically increase the apparent optical radius of the planet, and a hydrogen-rich envelope would not have survived erosion at such close distance from the host star over $\sim 10 \mathrm{~Gy}$. Instead, we find that a heavyweight atmosphere likely contributes to the planet radius, in agreement with recent results by Demory et al. (2016a) and Angelo \& Hu (2017). Degeneracies prevent us from assessing the composition of this envelope, which could include mineral-rich compounds arising from a molten or volcanic surface, or a $\mathrm{CO}$ - or $\mathrm{N}_{2}$-dominated atmosphere. In any case, the properties of the envelope would have to explain the temporal variability observed in the opacity of the planet. We compared all available measurements of $55 \mathrm{Cnc}$ e planet-to-star radius ratio to search for additional signatures of this variability, and found significant short-term ( $\sim$ week) variations in the STIS measurements. All measured sizes are nonetheless consistent within $3 \sigma$ with our derived radius, and we found no evidence for long-term variations over timescales of months or years. Observations at high photometric precision and high temporal cadence (e.g., with the CHEOPS satellite), along with high-resolution spectroscopic follow up, will be required to further investigate the variable nature of 55 Cnce.

Acknowledgements. We thank the referee for their constructive comments. We thank Munazza K. Alam and Mercedes Lopez-Morales for their help with the STIS/G750L observations, and N. Hara and P.A. Wilson for helpful discussions about correlated noise. This work has been carried out in the frame of the National Centre for Competence in Research "PlanetS" supported by the Swiss National Science Foundation (SNSF). V.B. acknowledges the financial support of the SNSF. X.D. is grateful to The Branco Weiss Fellowship Society in Science for continuous support. C.D. is funded by the Swiss National Science Foundation under the Ambizione grant PZ00P2_174028. This project has received funding from the European Research Council (ERC) under the European Union's Horizon 2020 research and innovation program (project Four Aces grant agreement No 724427). G.W.H. acknowledges long-term support from NASA, NSF, Tennessee State University, and the State of Tennessee through its Centers of Excellence program. N.A-D. acknowledges support from FONDECYT 3180063. We thank the Programme National de Planétologie for the use of SOPHIE at Observatoire de Haute Provence.

\section{References}

Angelo, I., \& Hu, R. 2017, AJ, 154, 232

Baluev, R. V. 2015, MNRAS, 446, 1493

Bouchet, J., Mazevet, S., Morard, G., Guyot, F., \& Musella, R. 2013, Phys. Rev. B, 87, 094102

Bourrier, V., \& Hébrard, G. 2014, A\&A, 569, A65

Bourrier, V., Lecavelier des Etangs, A., \& Vidal-Madjar, A. 2014, A\&A, 565, A105

Bourrier, V., Ehrenreich, D., Lecavelier des Etangs, A., et al. 2018, A\&A, 615, A117

Brewer, J. M., Fischer, D. A., Valenti, J. A., \& Piskunov, N. 2016, ApJS, 225, 32 Brown, T. M., Charbonneau, D., Gilliland, R. L., Noyes, R. W., \& Burrows, A. 2001, ApJ, 552, 699

Butler, R. P., Marcy, G. W., Williams, E., Hauser, H., \& Shirts, P. 1997, ApJ 474, L115

Butler, R. P., Vogt, S. S., Laughlin, G., et al. 2017, AJ, 153, 208

Connolly, J. A. D. 2009, Geochem, Geoph, Geosys, 10, Q10014

Crida, A., Ligi, R., Dorn, C., \& Lebreton, Y. 2018, ApJ, 860, 122

Dawson, R. I., \& Fabrycky, D. C. 2010, ApJ, 722, 937

de Mooij, E. J. W., López-Morales, M., Karjalainen, R., Hrudkova, M., \& Jayawardhana, R. 2014, ApJ, 797, L21

Demory, B.-O., Gillon, M., Deming, D., et al. 2011, A\&A, 533, A114

Demory, B.-O., Gillon, M., Seager, S., et al. 2012, ApJ, 751, L28

Demory, B.-O., Ehrenreich, D., Queloz, D., et al. 2015, MNRAS, 450, 2043

Demory, B.-O., Gillon, M., de Wit, J., et al. 2016a, Nature, 532, 207

Demory, B.-O., Gillon, M., Madhusudhan, N., \& Queloz, D. 2016b, MNRAS, 455, 2018

Dorn, C., Hinkel, N. R., \& Venturini, J. 2017a, A\&A, 597, A38

Dorn, C., Venturini, J., Khan, A., et al. 2017b, A\&A, 597, A37

Dragomir, D., Matthews, J. M., Eastman, J. D., et al. 2013, ApJ, 772, L2

Dragomir, D., Matthews, J. M., Winn, J. N., \& Rowe, J. F. 2014, in IAU Symp., ed. N. Haghighipour, 293, 52

Dumusque, X., Lovis, C., Ségransan, D., et al. 2011a, A\&A, 535, A55

Dumusque, X., Udry, S., Lovis, C., Santos, N. C., \& Monteiro, M. J. P. F. G. 2011b, A\&A, 525, A140

Eastman, J., Siverd, R., \& Gaudi, B. S. 2010, PASP, 122, 935

Eastman, J., Gaudi, B. S., \& Agol, E. 2013, PASP, 125, 83

Eaton, J. A., Henry, G. W., \& Fekel, F. C. 2003, Astrophys. Space Sci. Lib., 288, 189

Egeland, R., Soon, W., Baliunas, S., et al. 2017, ApJ, 835, 25

Ehrenreich, D., Bourrier, V., Bonfils, X., et al. 2012, A\&A, 547, A18

Elkins-Tanton, L. T. 2012, Ann. Rev. Earth Planet. Sci., 40, 113

Endl, M., Robertson, P., Cochran, W. D., et al. 2012, ApJ, 759, 19

Erkaev, N. V., Kulikov, Y. N., Lammer, H., et al. 2007, A\&A, 472, 329

Esteves, L. J., de Mooij, E. J. W., Jayawardhana, R., Watson, C., \& de Kok R. 2017, AJ, 153, 268

Evans, T. M., Pont, F., Sing, D. K., et al. 2013, ApJ, 772, L16

Fischer, D. A. 2017, 55 Cancri (Copernicus): A Multi-planet System with a Hot Super-Earth and a Jupiter Analogue, ed. B. Deeg H. (Cham, Switzerland: Springer)

Fischer, D. A., Marcy, G. W., Butler, R. P., et al. 2008, ApJ, 675, 790

Foreman-Mackey, D., Hogg, D. W., Lang, D., \& Goodman, J. 2013, PASP, 125, 306

Fortney, J. J., Marley, M. S., \& Barnes, J. W. 2007, ApJ, 659, 1661

Fulton, B. J., Petigura, E. A., Howard, A. W., et al. 2017, AJ, 154, 109 
Gillon, M., Demory, B.-O., Benneke, B., et al. 2012, A\&A, 539, A28

Ginzburg, S., Schlichting, H. E., \& Sari, R. 2016, ApJ, 825, 29

Gomes da Silva, J., Santos, N. C., Boisse, I., Dumusque, X., \& Lovis, C. 2014, A\&A, 566, A66

Hammond, M., \& Pierrehumbert, R. T. 2017, ApJ, 849, 152

Henry, G. W. 1995a, in Robotic Telescopes. Current Capabilities, Present Developments, and Future Prospects for Automated Astronomy, ed. G. W. Henry, \& J. A. Eaton, ASP Conf. Ser., 79, 44

Henry, G. W. 1995b, in Robotic Telescopes. Current Capabilities, Present Developments, and Future Prospects for Automated Astronomy, ed. G. W. Henry, \& J. A. Eaton, ASP Conf. Ser., 79, 37

Henry, G. W. 1999, PASP, 111, 845

Henry, G. W., Baliunas, S. L., Donahue, R. A., Fekel, F. C., \& Soon, W. 2000 ApJ, 531, 415

Holman, M. J., Winn, J. N., Latham, D. W., et al. 2006, ApJ, 652, 1715

Huitson, C. M., Sing, D. K., Vidal-Madjar, A., et al. 2012, MNRAS, 422, 2477

Ito, Y., Ikoma, M., Kawahara, H., et al. 2015, ApJ, 801, 144

Lecavelier des Etangs, A. 2007, A\&A, 461, 1185

Lopez, E. D. 2017, MNRAS, 472, 245

López-Morales, M., Triaud, A. H. M. J., Rodler, F., et al. 2014, ApJ, 792, L31

Lovis, C., Dumusque, X., Santos, N. C., et al. 2011, ArXiv e-prints [arXiv: 1107.5325]

Madhusudhan, N., Lee, K. K. M., \& Mousis, O. 2012, ApJ, 759, L40

Mamajek, E. E., \& Hillenbrand, L. A. 2008, ApJ, 687, 1264

Mandel, K., \& Agol, E. 2002, ApJ, 580, L171

Marcy, G. W., Butler, R. P., Fischer, D. A., et al. 2002, ApJ, 581, 1375

McArthur, B. E., Endl, M., Cochran, W. D., et al. 2004, ApJ, 614, L81

Meunier, N., Desort, M., \& Lagrange, A.-M. 2010a, A\&A, 512, A39
Meunier, N., Lagrange, A.-M., \& Desort, M. 2010b, A\&A, 519, A66 Motalebi, F., Udry, S., Gillon, M., et al. 2015, A\&A, 584, A72

Mugrauer, M., Neuhäuser, R., Mazeh, T., et al. 2006, Astron. Nachr., 327, 321

Nelson, B. E., Ford, E. B., Wright, J. T., et al. 2014, MNRAS, 441, 442

Noyes, R. W., Hartmann, L. W., Baliunas, S. L., Duncan, D. K., \& Vaughan, A. H. 1984, ApJ, 279, 763

Pont, F., Zucker, S., \& Queloz, D. 2006, MNRAS, 373, 231

Ridden-Harper, A. R., Snellen, I. A. G., Keller, C. U., et al. 2016, A\&A, 593, A129

Salz, M., Schneider, P. C., Czesla, S., \& Schmitt, J. H. M. M. 2016, A\&A, 585, L2

Seager, S., Kuchner, M., Hier-Majumder, C. A., \& Militzer, B. 2007, ApJ, 669, 1279

Sing, D. K., Vidal-Madjar, A., Désert, J.-M., Lecavelier des Etangs, A., \& Ballester, G. 2008, ApJ, 686, 658

Sing, D. K., Lecavelier des Etangs, A., Fortney, J. J., et al. 2013, MNRAS, 436, 2956

Tsiaras, A., Rocchetto, M., Waldmann, I. P., et al. 2016, ApJ, 820, 99

Valenti, J. A., \& Fischer, D. A. 2005, ApJS, 159, 141

Vazan, A., Kovetz, A., Podolak, M., \& Helled, R. 2013, MNRAS, 434, 3283

von Braun, K., Boyajian, T. S., ten Brummelaar, T. A., et al. 2011, ApJ, 740, 49

Wilson, P. A., Sing, D. K., Nikolov, N., et al. 2015, MNRAS, 450, 192

Winn, J. N., Holman, M. J., Henry, G. W., et al. 2009, ApJ, 693, 794

Winn, J. N., Matthews, J. M., Dawson, R. I., et al. 2011, ApJ, 737, L18

Yee, S. W., Petigura, E. A., \& von Braun K. 2017, ApJ, 836, 77

Zechmeister, M., \& Kürster, M. 2009, A\&A, 496, 577

Zhang, X., \& Showman, A. P. 2017, ApJ, 836, 73 


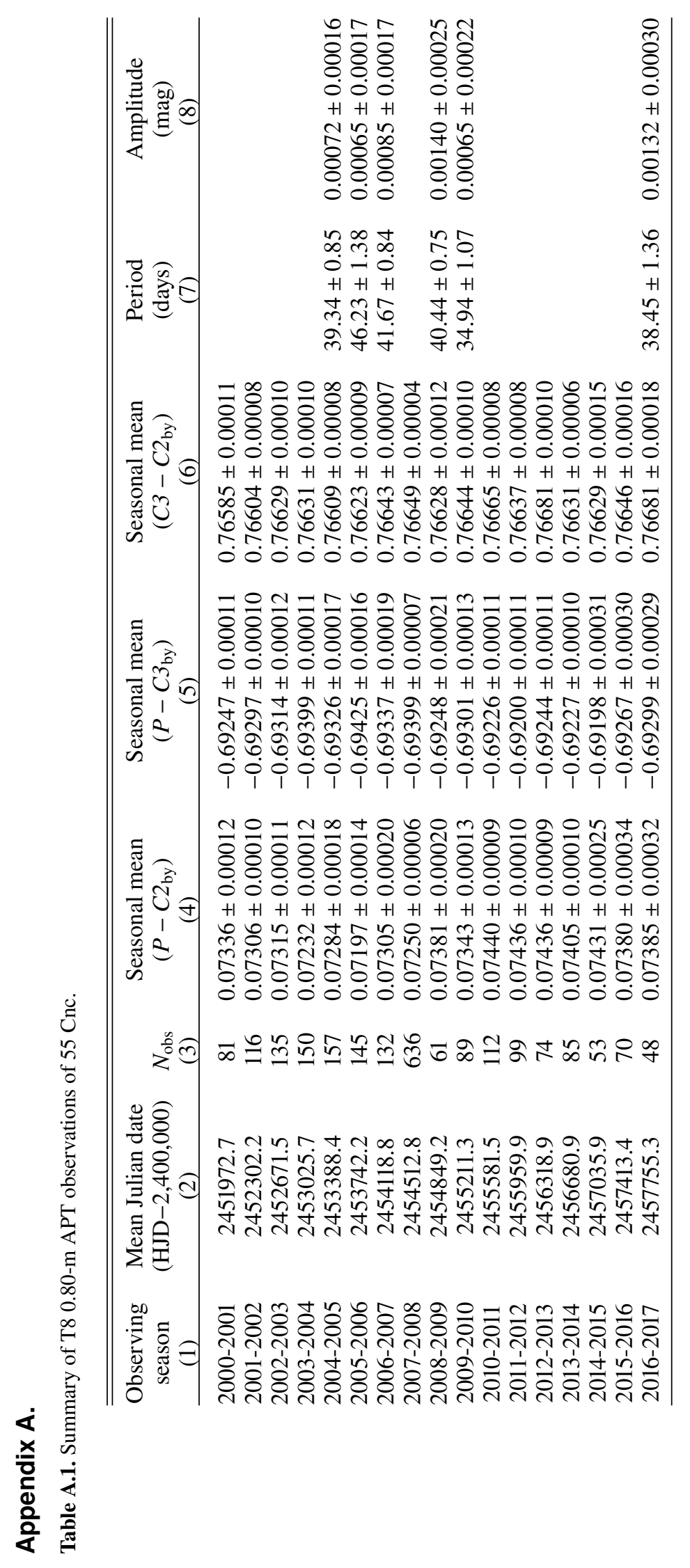


A\&A 619, A1 (2018)

Table A.2. Parameters probed by the MCMC used to fit the RV measurements of $55 \mathrm{Cnc}$.

\begin{tabular}{|c|c|c|c|c|c|c|c|c|c|c|}
\hline Param. & Units & $\operatorname{Max}($ Like) & Med & Mod & Std & $\mathrm{CI}(15.85)$ & $\mathrm{CI}(84.15)$ & $\mathrm{CI}(2.275)$ & $\mathrm{CI}(97.725)$ & Prior \\
\hline & & & & & & Likelihood & & & & \\
\hline $\log$ (Post) & & -2273.450488 & -2285.842126 & -2284.433522 & 4.255952 & -2290.966384 & -2281.311469 & -2297.110579 & -2277.307845 & \\
\hline $\log ($ Like $)$ & & -2291.046969 & -2302.472513 & -2302.414228 & 4.194558 & -2307.394292 & -2298.010475 & -2313.297187 & -2293.907193 & \\
\hline $\log$ (Prior) & & 17.596481 & 16.873550 & 17.204741 & 0.721226 & 15.862038 & 17.365749 & 14.233444 & 17.547586 & \\
\hline$M_{\star}$ & $\left(M_{\odot}\right)$ & 0.892695 & 0.904364 & 0.906670 & 0.013292 & 0.889584 & 0.919407 & 0.874257 & 0.935560 & $\mathcal{N}(0.905,0.015)$ \\
\hline$\sigma_{H A R P N}$ & $\left(\mathrm{~m} \mathrm{~s}^{-1}\right)$ & 1.06 & 1.34 & 1.18 & 0.26 & 1.09 & 1.66 & 0.89 & 2.10 & $\mathcal{u}$ \\
\hline$\sigma_{\text {HARPS }}$ & $\left(\mathrm{m} \mathrm{s}^{-1}\right)$ & 0.42 & 0.67 & 0.46 & 0.39 & 0.26 & 1.13 & 0.03 & 1.80 & $\mathcal{u}$ \\
\hline$\sigma_{H R S}$ & $\left(\mathrm{~m} \mathrm{~s}^{-1}\right)$ & 2.28 & 3.52 & 3.62 & 0.68 & 2.72 & 4.21 & 1.52 & 4.87 & $\mathcal{U}$ \\
\hline$\sigma_{K E C K}$ & $\left(\mathrm{~m} \mathrm{~s}^{-1}\right)$ & 2.91 & 3.35 & 3.30 & 0.21 & 3.12 & 3.59 & 2.86 & 3.85 & $\mathcal{u}$ \\
\hline$\sigma_{L I C K}$ & $\left(\mathrm{~m} \mathrm{~s}^{-1}\right)$ & 5.44 & 5.81 & 5.76 & 0.37 & 5.36 & 6.22 & 4.93 & 6.58 & $\mathcal{u}$ \\
\hline$\sigma_{S O P H I E}$ & $\left(\mathrm{~m} \mathrm{~s}^{-1}\right)$ & 1.69 & 1.95 & 1.93 & 0.28 & 1.66 & 2.29 & 1.39 & 2.69 & $\mathcal{u}$ \\
\hline$\sigma_{T U L L}$ & $\left(\mathrm{~m} \mathrm{~s}^{-1}\right)$ & 3.59 & 3.89 & 3.82 & 0.35 & 3.53 & 4.31 & 3.17 & 4.76 & $\mathcal{u}$ \\
\hline$\sigma_{J I T}$ & $\left(\mathrm{~m} \mathrm{~s}^{-1}\right)$ & 3.81 & 2.81 & 2.76 & 0.90 & 1.66 & 3.71 & 0.39 & 4.40 & $\mathcal{u}$ \\
\hline$\gamma_{H A R P N}$ & $\left(\mathrm{~m} \mathrm{~s}^{-1}\right)$ & 27451.45 & 27451.91 & 27451.85 & 0.89 & 27450.84 & 27452.89 & 27449.86 & 27453.93 & $\mathcal{u}$ \\
\hline$\gamma_{\text {HARPS }}$ & $\left(\mathrm{m} \mathrm{s}^{-1}\right)$ & 27468.87 & 27469.02 & 27468.75 & 0.82 & 27468.13 & 27469.97 & 27467.21 & 27470.93 & $\mathcal{u}$ \\
\hline$\gamma_{H R S}$ & $\left(\mathrm{~m} \mathrm{~s}^{-1}\right)$ & 28396.92 & 28397.31 & 28397.27 & 0.79 & 28396.39 & 28398.21 & 28395.53 & 28399.10 & $\mathcal{u}$ \\
\hline$\gamma_{K E C K}$ & $\left(\mathrm{~m} \mathrm{~s}^{-1}\right)$ & -40.91 & -40.89 & -40.95 & 0.40 & -41.34 & -40.42 & -41.75 & -39.92 & $\mathcal{u}$ \\
\hline$\gamma_{\text {LICK }}$ & $\left(\mathrm{m} \mathrm{s}^{-1}\right)$ & 3.43 & 3.74 & 3.60 & 0.41 & 3.28 & 4.22 & 2.83 & 4.72 & $\mathcal{u}$ \\
\hline$\gamma_{S O P H I E}$ & $\left(\mathrm{~m} \mathrm{~s}^{-1}\right)$ & 27437.78 & 27438.00 & 27437.85 & 0.81 & 27437.10 & 27438.96 & 27436.19 & 27439.84 & $\mathcal{u}$ \\
\hline$\gamma_{T U L L}$ & $\left(\mathrm{~m} \mathrm{~s}^{-1}\right)$ & -22571.04 & -22571.01 & -22571.27 & 0.57 & -22571.66 & -22570.35 & -22572.30 & -22569.68 & $\mathcal{u}$ \\
\hline $\log (P)$ & (d) & 1.165883 & 1.165885 & 1.165885 & 0.000002 & 1.165883 & 1.165887 & 1.165881 & 1.165889 & $\mathcal{u}$ \\
\hline $\log (K)$ & $\left(\mathrm{m} \mathrm{s}^{-1}\right)$ & 1.85 & 1.85 & 1.85 & 0.00 & 1.85 & 1.85 & 1.85 & 1.86 & $\mathcal{u}$ \\
\hline$\sqrt{e} \cdot \cos \omega$ & & 0.022977 & 0.031298 & 0.044307 & 0.031656 & -0.009855 & 0.064623 & -0.043345 & 0.086918 & $\mathcal{u}$ \\
\hline$\sqrt{e} \cdot \sin \omega$ & & -0.076941 & -0.006118 & -0.005375 & 0.030482 & -0.041314 & 0.030238 & -0.066334 & 0.058214 & $\mathcal{u}$ \\
\hline$\lambda_{0}$ & (deg) & 198.337280 & 198.236638 & 198.254369 & 0.170587 & 198.040883 & 198.429919 & 197.842096 & 198.617500 & $\mathcal{u}$ \\
\hline $\log (P)$ & (d) & 1.647371 & 1.647372 & 1.647365 & 0.000036 & 1.647330 & 1.647413 & 1.647286 & 1.647457 & $\mathcal{u}$ \\
\hline $\log (K)$ & $\left(\mathrm{m} \mathrm{s}^{-1}\right)$ & 0.99 & 1.00 & 0.99 & 0.01 & 0.99 & 1.00 & 0.98 & 1.01 & $\mathcal{u}$ \\
\hline$\sqrt{e} \cdot \cos \omega$ & & 0.145067 & 0.139330 & 0.154716 & 0.082223 & 0.025951 & 0.214780 & -0.079089 & 0.266234 & $\mathcal{u}$ \\
\hline$\sqrt{e} \cdot \sin \omega$ & & -0.021607 & 0.007209 & 0.019499 & 0.083988 & -0.092205 & 0.104252 & -0.174251 & 0.175082 & $\mathcal{u}$ \\
\hline$\lambda_{0}$ & (deg) & 152.107645 & 152.036764 & 151.667729 & 1.009441 & 150.913959 & 153.195011 & 149.746100 & 154.276811 & $\mathcal{u}$ \\
\hline $\log (P)$ & (d) & 2.414600 & 2.414770 & 2.414529 & 0.000433 & 2.414285 & 2.415259 & 2.413794 & 2.415791 & $\mathcal{u}$ \\
\hline $\log (K)$ & $\left(\mathrm{m} \mathrm{s}^{-1}\right)$ & 0.73 & 0.71 & 0.71 & 0.02 & 0.69 & 0.73 & 0.66 & 0.75 & $\mathcal{u}$ \\
\hline$\sqrt{e} \cdot \cos \omega$ & & 0.028362 & -0.029096 & -0.132560 & 0.133065 & -0.187318 & 0.130365 & -0.294743 & 0.249554 & $\mathcal{u}$ \\
\hline$\sqrt{e} \cdot \sin \omega$ & & -0.270494 & -0.225158 & -0.278934 & 0.113215 & -0.322547 & -0.067598 & -0.392342 & 0.091226 & $\mathcal{u}$ \\
\hline$\lambda_{0}$ & (deg) & 106.069275 & 102.615196 & 103.432101 & 2.715291 & 99.345207 & 105.648564 & 96.345607 & 108.304627 & $\mathcal{u}$ \\
\hline $\log (P)$ & (d) & 3.580323 & 3.582340 & 3.581415 & 0.007485 & 3.573450 & 3.590936 & 3.565279 & 3.598119 & $\mathcal{u}$ \\
\hline $\log (K)$ & $\left(\mathrm{m} \mathrm{s}^{-1}\right)$ & 1.18 & 1.18 & 1.19 & 0.05 & 1.13 & 1.22 & 1.06 & 1.27 & $\mathcal{u}$ \\
\hline$\sqrt{e} \cdot \cos \omega$ & & -0.391429 & -0.400855 & -0.384878 & 0.043763 & -0.455229 & -0.354370 & -0.499443 & -0.309570 & $\mathcal{U}$ \\
\hline$\sqrt{e} \cdot \sin \omega$ & & 0.049300 & 0.038499 & 0.065933 & 0.094395 & -0.079834 & 0.137095 & -0.186755 & 0.227198 & $\mathcal{u}$ \\
\hline$\lambda_{0}$ & (deg) & 122.695667 & 124.709302 & 126.275021 & 5.071957 & 119.011467 & 130.603288 & 114.471907 & 137.366403 & $\mathcal{u}$ \\
\hline $\log (P)$ & (d) & 3.749135 & 3.746185 & 3.745006 & 0.006227 & 3.739227 & 3.753435 & 3.733291 & 3.760178 & $\mathcal{U}$ \\
\hline $\log (K)$ & $\left(\mathrm{m} \mathrm{s}^{-1}\right)$ & 1.58 & 1.59 & 1.59 & 0.01 & 1.57 & 1.60 & 1.56 & 1.61 & $\mathcal{u}$ \\
\hline$\sqrt{e} \cdot \cos \omega$ & & 0.134776 & 0.128560 & 0.127138 & 0.043737 & 0.081188 & 0.180318 & 0.025802 & 0.223687 & $\mathcal{u}$ \\
\hline$\sqrt{e} \cdot \sin \omega$ & & -0.328459 & -0.334039 & -0.346717 & 0.031095 & -0.368411 & -0.298759 & -0.403605 & -0.261516 & $\mathcal{u}$ \\
\hline$\lambda_{0}$ & (deg) & 7.523549 & 8.373486 & 7.475054 & 3.442002 & 4.614703 & 12.050153 & 0.232590 & 15.802723 & $\mathcal{u}$ \\
\hline$P$ & (d) & 0.736546 & 0.736547 & 0.736547 & 0.000001 & 0.736546 & 0.736549 & 0.736545 & 0.736550 & $\mathcal{N}(0.7365462780,1.8477 e-06)$ \\
\hline $\log (K)$ & $\left(\mathrm{m} \mathrm{s}^{-1}\right)$ & 0.77 & 0.78 & 0.78 & 0.01 & 0.76 & 0.80 & 0.74 & 0.81 & $\mathcal{U}$ \\
\hline$\sqrt{e} \cdot \cos \omega$ & & 0.065426 & 0.012710 & -0.019946 & 0.079210 & -0.083201 & 0.105674 & -0.160391 & 0.174811 & $\mathcal{u}$ \\
\hline$\sqrt{e} \cdot \sin \omega$ & & 0.234745 & 0.192120 & 0.220349 & 0.093920 & 0.063752 & 0.268215 & -0.076741 & 0.331372 & $\mathcal{u}$ \\
\hline$T_{C}$ & (d) & 55733.005597 & 55733.005980 & 55733.006299 & 0.001265 & 55733.004556 & 55733.007423 & 55733.003120 & 55733.008795 & $\mathcal{N}(55733.0058594,0.0014648)$ \\
\hline
\end{tabular}

Notes. The maximum likelihood solution (Max(Like), the median (Med), mode (Mod), and standard deviation (Std) of the posterior distribution for each parameter is shown, as well as the $68.3 \%(\mathrm{CI}(15.85), \mathrm{CI}(84.15)$ and $95.45 \%(\mathrm{CI}(2.275), \mathrm{CI}(97.725)$ confidence intervals. The prior for each parameter can be of type: $\mathcal{U}$ : uniform, $\mathcal{N}$ : normal, $\mathcal{S N}$ : split normal, or $\mathcal{T} \mathcal{N}$ : truncated normal. 